\title{
A MASSIVE PROTOSTAR EMBEDDED IN THE SCUBA CORE JCMT 18354-0649S
}

\author{
Ming Zhu ${ }^{1}$, C. J. Davis ${ }^{2}$, Yufang Wu ${ }^{3}$, B. A. Whitney ${ }^{4}$, T. Robitaille ${ }^{5}$, And R. Peng ${ }^{6}$ \\ ${ }^{1}$ National Astronomical Observatory of China, 20A Datun Road, Chaoyang District, Beijing, China; mz@nao.cac.cn \\ 2 Joint Astronomy Centre, 660 N. A'ohoku Place, Hilo, HI 96720, USA \\ ${ }^{3}$ Astronomy Department, Peking University, Beijing 100871, China \\ ${ }^{4}$ Space Science Institute, 4750 Walnut Street, Suite 205, Boulder, CO 80301, USA \\ ${ }^{5}$ Harvard-Smithsonian Center for Astrophysics, 60 Garden Street, Cambridge, MA 02138, USA \\ ${ }^{6}$ Caltech Submillimeter Observatory, 111 Nowelo Street, Hilo, HI 96720, USA \\ Received 2010 September 17; accepted 2011 June 30; published 2011 September 6
}

\begin{abstract}
We report the discovery of an extremely red object embedded in the massive SCUBA core JCMT 18354-0649S. This object is not associated with any known radio or far-IR source, though it appears in Spitzer IRAC data obtained as part of the GLIMPSE survey. At shorter wavelengths, this embedded source exhibits an extreme color, $K-L^{\prime}=$ 6.7. At an assumed distance of $5.7 \mathrm{kpc}$, this source has a near-IR luminosity of $\sim 1000 L_{\odot}$. Its spectral energy distribution (SED) rises sharply from $2.1 \mu \mathrm{m}$ to $8 \mu \mathrm{m}$, similar to that of a Class 0 young stellar object. Theoretical modeling of the SED indicates that the central star has a mass of 6-12 $M_{\odot}$, with an optical extinction of more than 30. As both inflow and outflow motions are present in JCMT 18354-0649S, we suggest that this deeply embedded source is (1) a massive protostar in the early stages of accretion, and (2) the driving source of a massive molecular outflow evident in HCN $J=3-2$ profiles observed toward this region.
\end{abstract}

Key words: infrared: ISM - infrared: stars - stars: formation

Online-only material: color figures

\section{INTRODUCTION}

Massive stars play an essential role in galaxy evolution, but the study of massive star formation (MSF) lags far behind that of low-mass stars. A major question is whether massive stars form through accretion and outflow mechanisms like low-mass stars (McKee \& Tan 2003), or via coalescence and collision (Bonnell et al. 1998). Tests of these two hypotheses are hampered by the lack of observations of a sufficient number of high-mass protostellar objects (HMPOs), sources that are precursors to ultracompact $\mathrm{H}$ II regions and are analogous to low-mass Class 0 sources (Andre et al. 2000). To date, only a few HMPO candidates have been identified in the literature (e.g., Davis et al. 2004; Shepherd et al. 2004; Rathborne et al. 2005; Beuther \& Shepherd 2005; Beuther et al. 2002, 2007, and reference therein). Detailed studies of these and other objects can reveal the physical conditions of massive protostars at their earliest stages.

We have conducted a series of observations using the James Clerk Maxwell Telescope (JCMT) to search for HMPOs in a sample of massive ammonia cores that are not associated with IRAS sources. JCMT 18354-0649N and JCMT 183540649S (hereafter J18354N and J18354S) are two SCUBA cores separated by $\sim 1^{\prime}$ on the sky (see Figure 1 in Section 3 ). The southern source, J18354S, has the hallmarks of an HMPO and is the first such candidate found in our survey ( $\mathrm{Wu}$ et al. 2005, 2006). J18354N coincides with the known H II region G25.4NW (Lester et al. 1985); the southern core has no known radio, IRAS, or $M S X$ sources associated with it. J18354S has a diameter of $\sim 10^{\prime \prime}$, corresponding to $0.28 \mathrm{pc}$ at an assumed distance of $5.7 \mathrm{kpc}$. The total mass of J18354S is $820 M_{\odot}$ and the average density is $1.1 \times 10^{6} \mathrm{~cm}^{-3}$ in a region with a radius of $5^{\prime \prime}$. However, this source is located in the galactic plane $(l=25.3852, b=-0.1466)$ and the distance ambiguity implies that the distance could be 5.7 or $9.6 \mathrm{kpc}$, depending on whether it is located at the near or far side of the spiral arm. If the distance is the latter, the source would be even more massive.

Spectral line observations of high-density tracers, including $\mathrm{HCN}(3-2), \mathrm{HCO}^{+}(3-2), \mathrm{H}^{13} \mathrm{CO}^{+}(3-2)$, and $\mathrm{C}^{17} \mathrm{O}(2-1)$, reveal a prominent blue profile (Wu et al. 2005), which was confirmed with the Submillimeter Array (SMA) observations (Liu et al. 2011), indicating that this core is collapsing. In addition, at the full width zero intensity (FWZI) level the $\mathrm{HCN}(3-2)$ and $\mathrm{CO}(3-2)$ line profiles span $38 \mathrm{~km} \mathrm{~s}^{-1}$ (Wu et al. 2005), indicating outflow motions in this region. The age of the outflow was estimated to be less than 6600 years and the mass infall rate is $3.4 \times 10^{-3} M_{\odot} \mathrm{yr}^{-1}$, so up to $10-20 M_{\odot}$ could have been accumulated in the core (Wu et al. 2005).

In this paper we present follow-up observations in the nearinfrared (near-IR) $H, K$, and $L^{\prime}$ bands. An extremely red object was discovered at the center of the SCUBA core (hereby called IRS1a, see Section 3.2). We have also used the Caltech Submillimeter Observatory (CSO) to map the $350 \mu \mathrm{m}$ dust emission, and the JCMT to observe the $\mathrm{HCN}(4-3), \mathrm{CO}(3-2)$, $\mathrm{C}^{17} \mathrm{O}(3-2), \mathrm{H}_{2} \mathrm{CO}(3-2)$, and $\mathrm{CH}_{3} \mathrm{OH}(7-6)$ lines to probe the star formation environment in this region. The observational details and data analysis are presented in the following sections.

\section{OBSERVATIONS AND DATA REDUCTION}

\subsection{UKIRT Data}

Images of the JCMT 18354-0649 region were obtained at the United Kingdom Infrared Telescope (UKIRT) on 2004 September 17 and 19, and on 2007 June 27, using the UIST imagerspectrometer (Ramsay Howatt et al. 2004) under photometric conditions. UIST employs a $1024 \times 1024$ pixel Aladin II InSb array. $H(\lambda=1.64 \mu \mathrm{m}, \Delta \lambda=0.29 \mu \mathrm{m})$ and $K(\lambda=2.20 \mu \mathrm{m}$, $\Delta \lambda=0.34 \mu \mathrm{m})$ band images were acquired with a plate scale of $0^{\prime \prime} 12$ pixel $^{-1} ; L^{\prime}(\lambda=3.77 \mu \mathrm{m}, \Delta \lambda=0.68 \mu \mathrm{m})$ band images were obtained with a 0.06 pixel $^{-1}$ plate scale. We also used a 


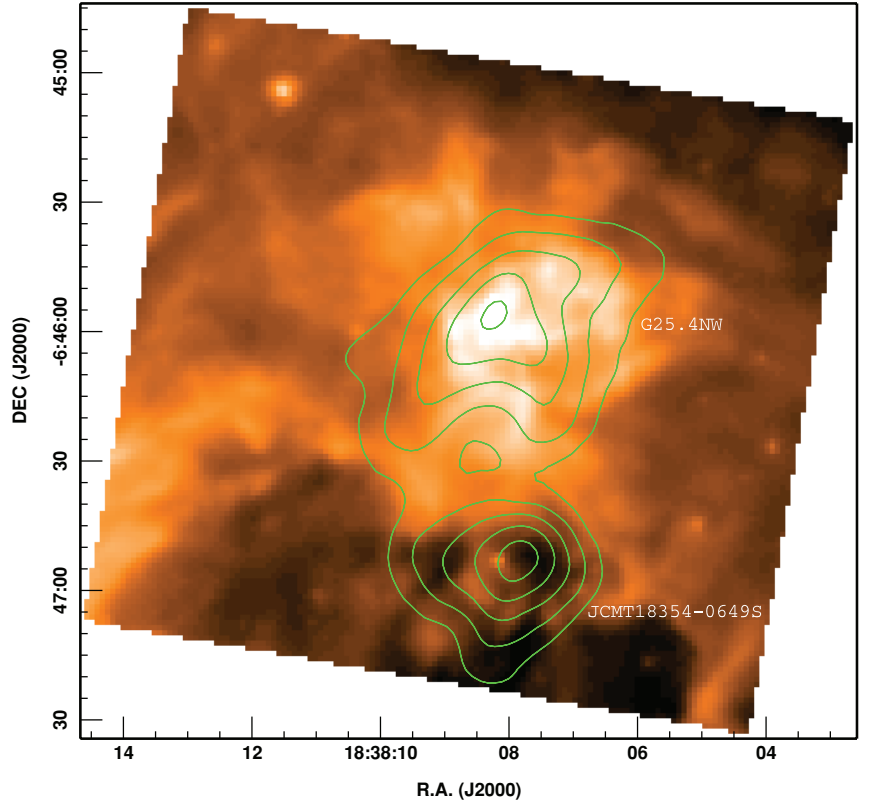

Figure 1. Spitzer and CSO observations of the two SCUBA cores JCMT $18354-0649 \mathrm{~N}$ and JCMT 18354-0649S. The two cores are evident in the CSO $350 \mu \mathrm{m}$ data (contours), though only the northern core is seen in the IRAC $8 \mu \mathrm{m}$ image. Note that JCMT 18354-0649N coincides with a well-known H II region G25.4NW. The distance between the two cores is approximately $1.5 \mathrm{pc}$. The contour levels are 10,14.1, 20, 28.2, and $40 \mathrm{Jy} \mathrm{beam}^{-1}$ and image sensitivity is $1 \sigma=0.8 \mathrm{Jy} \mathrm{beam}^{-1}$.

(A color version of this figure is available in the online journal.)

narrowband filter $(\lambda=2.122 \mu \mathrm{m}, \Delta \lambda=0.031 \mu \mathrm{m})$ to search for $\mathrm{H}_{2}$ 1-0 S(1) rovibrational line emission.

Nine-point "jittered" mosaics were obtained at the shorter near-IR wavelengths, resulting in a field of view of $160^{\prime \prime} \times 160^{\prime \prime}$ in the reduced mosaic. An eight-point jitter pattern was used at $3.8 \mu \mathrm{m}$ (chopping was not necessary) to give coverage over an area of $60^{\prime \prime} \times 78^{\prime \prime}$. The total per-pixel on-source integration time amounted to 27 minutes, 18 minutes, and 34 minutes in the $H$-, $K$-, and $L^{\prime}$-band mosaics, respectively. In addition, 10 minutes of integration time were acquired with the $2.12 \mu \mathrm{m}$ narrowband filter.

Standard data reduction techniques were employed, involving dark subtraction and bad-pixel masking together with flatfielding and (at $L^{\prime}$ ) sky subtraction, using the source frames themselves. The $O R A C-D R$ pipeline software was used for this task. Images were subsequently registered by hand using field stars and the Starlink CCDPack suite of software. The "seeing" in the reduced mosaics (the average FWHM intensity measured from two-dimensional Gaussian fits to field stars) measures $0.6-0.7$ at $H$ and $K$, and 0.55 at $L^{\prime}$. The UKIRT standards FS 35 and HD 161903 were used for flux calibration.

\subsection{Spitzer IRAC Data}

The Spitzer $3.5-8.0 \mu \mathrm{m}$ images were obtained as part of the Galactic Legacy Infrared Mid-Plane Survey Extraordinaire (GLIMPSE; Benjamin et al. 2003) using the Infrared Array Camera (IRAC; Fazio et al. 2004). The IRAC data were processed by the GLIMPSE team; image mosaics were created using the GLIMPSE pipeline (v10) after artifacts such as cosmic rays, stray light, column pull-down, and banding had been removed. ${ }^{7}$ We have downloaded the images and confirmed the

\footnotetext{
7 Details of the data processing can be found at http://www.astro.wisc.edu/ glimpse/docs.html.
}

calibration by comparing our flux measurements with those listed in the GLIMPSE catalog.

The $24 \mu \mathrm{m}$ and $70 \mu \mathrm{m}$ data from the Multi-band Imaging Photometer for Spitzer (MIPS) were also downloaded from the Spitzer archive. The data were originally taken as part of the MIPSGAL survey (Carey et al. 2005) and have been processed using the Spitzer Science Center data reduction pipeline.

The near-infrared (NIR) and mid-infrared (MIR) photometries were performed using the GAIA tools from the Starlink package. For Spitzer IRAC data, we followed the procedure in the IRAC instrument handbook. ${ }^{8}$ The $\mathrm{J} 18354 \mathrm{~S}$ regions have significant diffused interstellar emission; especially in IRAC channels 3 and 4, where the interstellar polycyclic aromatic hydrocarbon (PAH) bands have highly structured emission; thus we choose to use a circular aperture of four native pixels (4".8) in diameter, and aperture correction was performed based on the factors in Table 4.7 of the handbook. The flux density was obtained by integrating over the source, and the area-corrected background was subtracted. However, due to variation in the background emission in the J18354S area, uncertainty in the determination of the background level is a dominant contributor to the overall uncertainty in the measured flux densities. We used a separate aperture to measure the local background, and care was taken in placing the background regions to avoid areas with emission from nearby sources. We have also measured different background regions to estimate the variation in the background emission level. The uncertainty in the resulting fluxes is estimated to be about $20 \%$ in the IRAC 3.6 and $4.5 \mu \mathrm{m}$ bands and about $25 \%$ in the IRAC 5.8 and $8.0 \mu \mathrm{m}$ bands.

\subsection{CSO Observations}

Observations at $350 \mu \mathrm{m}$ were made on two occasions with the second-generation Submillimeter High Angular Resolution Camera (SHARC II; Dowell et al. 2003) at the CSO on 2006 July 7 and September 1, respectively. On each occasion, eight scans of 4 minute duration were obtained, covering a rectangular area of $3.5 \times 5.5$ in azimuth and elevation. Neptune and Uranus were used as primary flux calibrators; 3 minute scans were made before and after observations of the science target. The telescope pointing, checked on an hourly basis, was found to be better than 3". The Dish Surface Optimization System (DSOS; Leong et al. 2006) was active during the observations to correct for deformation of the telescope surface due to gravity.

Data reduction was conducted with CRUSH, a Java-based application developed specifically for SHARC II. The final image of the JCMT 18354-0649 region was made by combining all of the scans with proper flux calibration. This yielded a smoothed FWHM of 9!' 4 and an image sensitivity of $\sim 0.8 \mathrm{Jy} \mathrm{beam}^{-1}$. Major uncertainties came from atmospheric correction and flux calibration, which was estimated to be about $20 \%$. Thus, the absolute scale of the fluxes could have an error of up to $20 \%$.

\subsection{JCMT Observations}

In order to probe the physical conditions of the SCUBA core J18354S, we used the JCMT to observe a number of molecular species and transitions. Table 1 lists the detailed observational parameters for each molecular spectral line observed. These observations were mostly conducted by ourselves during several observing runs, though some data were taken in flexible observing mode. In all cases we used the "position switch" observing

\footnotetext{
8 http://ssc.spitzer.caltech.edu/irac/iracinstrumenthandbook/32/.
} 
Table 1

A List of the Lines and Frequencies Observed with the JCMT

\begin{tabular}{llcccc}
\hline \hline Line & Date & Receiver & $\begin{array}{c}\text { Freq } \\
(\mathrm{GHz})\end{array}$ & $\begin{array}{c}\text { HPBW } \\
(\operatorname{arcsec})\end{array}$ & Map-type \\
\hline $\mathrm{HCN}(4-3)$ & 2005 Oct & RXB & 354.51 & 14.6 & $5 \times 5$ grid \\
${ }^{12} \mathrm{CO}(3-2)$ & 2005 Aug & RXB & 345.79 & 14.6 & $5 \times 5$ grid \\
$\mathrm{C}^{17} \mathrm{O}(3-2)$ & 2004 Oct & RXB & 337.06 & 14.6 & $3 \times 3$ grid \\
$\mathrm{CH}_{3} \mathrm{OH}(7-6)$ & 2006 Feb & RXB & 338.41 & 14.6 & $5 \times 5$ grid \\
$\mathrm{C}^{17} \mathrm{O}(2-1)$ & 2004 Jun & RXA & 224.71 & 21.3 & $3 \times 3$ grid \\
$\mathrm{C}^{18} \mathrm{O}(2-1)$ & 2004 May & RXA & 219.56 & 21.3 & Raster \\
$\mathrm{SiO}(6-5)$ & 2004 Jun & RXA & 260.52 & 19.0 & 2 points \\
$\mathrm{H}_{2} \mathrm{CO}\left(3_{03}-202\right)$ & 2004 Jun & RXA & 218.37 & 21.3 & 2 points \\
\hline
\end{tabular}

mode and employed the Digital Autocorrelation Spectrometer, with a bandwidth of $250 \mathrm{MHz}$, as the back end. This provided a velocity resolution of $0.25 \mathrm{~km} \mathrm{~s}^{-1}$ for the A-band receiver (RXA) and $0.38 \mathrm{~km} \mathrm{~s}^{-1}$ for the $\mathrm{B}$-band receiver (RXB). The measured main-beam efficiencies $\left(\eta_{\mathrm{mb}}\right)$ were 0.69 for RXA and 0.63 for RXB, with $15 \%$ uncertainty. The pointing was checked regularly using G34.3; we estimate an rms pointing uncertainty of $\delta \theta_{\text {rms }} \sim 2^{\prime \prime}-3^{\prime \prime}$.

The data were reduced using the Starlink package SPECX; linear baselines were subtracted from all of the spectra.

\section{RESULTS}

\subsection{The Dust Cores, J18354N and J18354S, in JCMT 18354-0649}

Figure 1 presents our CSO $350 \mu \mathrm{m}$ observations, overlaid as contours on a Spitzer IRAC $8 \mu \mathrm{m}$ image of the region. Both cores are clearly detected (note that $\mathrm{J} 18354 \mathrm{~N}$ coincides with $\mathrm{G} 25.4 \mathrm{NW}$ ). The morphologies of the two $350 \mu \mathrm{m}$ peaks are consistent with what is seen in the 450 and $850 \mu \mathrm{m}$ maps published by $\mathrm{Wu}$ et al. (2005). At these wavelengths the southern core appears to be more compact and brighter (with a higher peak intensity) than the northern core, although the total submillimeter fluxes are not significantly different between the two cores. The northern core is a known H II region, G25.4NW (Lester et al. 1985). It is heated by a cluster of OB stars with a total far-IR luminosity of more than $5 \times 10^{5} L_{\odot}$. It has the same radial velocity as $\mathrm{J} 18354 \mathrm{~S}$ and hence is presumably at a similar distance. By comparing the dust and gas properties of these two cores, we will show that the southern core has the characteristics of a massive star-forming region at an earlier stage in its evolution.

If the dust emission is optically thin, in theory we can combine the 350,450 , and $850 \mu \mathrm{m}$ fluxes to derive the dust temperature and emissivity index, $\beta$, using the following formulae (Zhu et al. 2009):

$$
\begin{aligned}
& \frac{S_{450}}{S_{850}}=1.88^{\beta+3}\left(\frac{e^{16.8 / T}-1}{e^{31.8 / T}-1}\right) \\
& \frac{S_{350}}{S_{850}}=2.43^{\beta+3}\left(\frac{e^{16.8 / T}-1}{e^{40.8 / T}-1}\right) .
\end{aligned}
$$

The highest optical depth for the submillimeter emission is at the center of the dust core. As an estimate, assuming a dust temperature of $20 \mathrm{~K}, \beta=2$, and a core size of $10^{\prime \prime}$, the optical depth at the core center of J18354S would be $\sim 0.05,0.18$, and 0.34 at 850,450 , and $350 \mu \mathrm{m}$, respectively. Thus, it is safe to assume that the core center is optically thin at $850 \mu \mathrm{m}$. Optical effects need to be considered at the core center at short wavelengths: we need to apply a factor of $\left(1-\tau_{\nu} / 2\right)$ in Equations
Table 2

Submillimeter Fluxes and Fitting Results for the Dust Cores in JCMT 18354-0649

\begin{tabular}{lccccccc}
\hline \hline Name & $\begin{array}{c}350 \mu \mathrm{m} \\
(\mathrm{Jy})\end{array}$ & $\begin{array}{c}450 \mu \mathrm{m} \\
(\mathrm{Jy})\end{array}$ & $\begin{array}{c}850 \mu \mathrm{m}^{\mathrm{a}} \\
(\mathrm{Jy})\end{array}$ & $\beta$ & $\begin{array}{c}T_{\mathrm{d}} \\
(\mathrm{K})\end{array}$ & $\begin{array}{c}M_{\text {dust }} \\
\left(M_{\odot}\right)\end{array}$ & $\begin{array}{c}\text { Aperture } \\
(\operatorname{arcsec})\end{array}$ \\
\hline $\mathrm{J} 18354 \mathrm{~S}$ & $91 \pm 25$ & $39 \pm 9.5$ & $4.3 \pm 0.9$ & $2.3 \pm 0.4$ & $18 \pm 4$ & 670 & 15 \\
$\mathrm{~J} 18354 \mathrm{~N}$ & $86 \pm 25$ & $31 \pm 7.5$ & $3.7 \pm 0.9$ & $2.0 \pm 0.4$ & $24.5 \pm 4$ & 450 & 15
\end{tabular}

Note. ${ }^{\text {a }}$ The $850 \mu \mathrm{m}$ fluxes have been corrected for $\mathrm{CO}(3-2)$ flux contamination, which is about 0.21 and $0.38 \mathrm{Jy}$ for the southern and northern cores, respectively.

(1) and (2), where $\tau_{v}$ is the optical depth at 450 and $350 \mu \mathrm{m}$, respectively.

In Figure 2 we show (a) $350 \mu \mathrm{m} / 450 \mu \mathrm{m}$ and (b) $350 \mu \mathrm{m} /$ $850 \mu \mathrm{m}$ ratio maps. The data used to construct each map have been smoothed with a Gaussian function to the same resolution: 9."4 in (a) and $15^{\prime \prime}$ in (b). The lowest flux ratios are measured toward the two cores, particularly J18354S, which stands out as having the very lowest ratios. One would expect this to be the case if this core is associated with large amounts of cold dust (we assume that $\beta$ does not vary across the region). The cold spots in the northern $\mathrm{H}_{\mathrm{II}}$ region are presumably associated with parts of the cloud that have not been completely heated through by the massive stars in the $\mathrm{H}$ II region (a similar situation exists in the Orion Bar; Johnstone \& Bally 1999). Higher ratios are measured in the warmer envelopes that surround each core. Note, however, that the fluxes in the outer regions (about 15" away from the core center) in each map have large uncertainties. This is mostly due to poor signal-to-noise ratios and side lobe structures that are not well understood, especially at 450 and $350 \mu \mathrm{m}$. Also, there could be missing flux in the SCUBA data at 450 and $850 \mu \mathrm{m}$ due to chopping. These errors may result in unrealistically high $350 / 450$ and $350 / 850$ ratios.

More quantitative estimates of the dust temperature, $T_{\mathrm{d}}$, and emissivity index, $\beta$, are listed in Table 2 . We define the regions in the cores $\left(r<7^{\prime \prime} .5\right)$ and the envelope $\left(7^{\prime \prime} .5<r<20^{\prime \prime}\right)$ for J18354S. The northern H II region, J18354N/G25.4NW, does not have a clear central core. Thus, we list values for the central $15^{\prime \prime}$ (diameter), for comparison. We find that $\beta=2.0 \pm 0.4$ in $\mathrm{J} 18354 \mathrm{~N}$ and $\beta=2.3 \pm 0.4$ in $\mathrm{J} 18354 \mathrm{~S}$. The latter is higher than the values found in many HMPOs where $\beta \sim 1$ (Williams et al. 2004; Beuther \& Steinacker 2007), in some molecular cores where $\beta \sim 1.6$ (Friesen et al. 2005), and in most giant molecular clouds where typically $\beta \leqslant 2$ (cf. Goldsmith et al. 1997 and references therein), but it is still consistent with some massive star-forming regions, such as the Orion $\operatorname{Bar}(\beta=2.5$; Lis et al. 1998) and GCM $0.25+0.11(\beta=2.8$; Lis \& Menten 1998). The gradient in $\beta$ in the JCMT 18354-0649 region is consistent with the findings of Lis \& Menten (1998) who note that $\beta$ increases as the telescope beam moves away from the Orion Bar, a photon-dominated region, toward more quiescent gas further north. A larger $\beta$ in quiescent clouds is usually attributed to the growth of ice mantles on grains, such as in the starless cores TMC-1 (Schnee et al. 2010). However, the value of $\beta$ carries a large uncertainty, mainly due to the uncertainties associated with the submillimeter fluxes. Thus, the variation in the emissivity index across the JCMT 18354-0649 region may not be significant.

For J18354S, the dust temperature is $18 \pm 4 \mathrm{~K}$ in the core (the central $15^{\prime \prime}$ in diameter) and $21 \pm 4 \mathrm{~K}$ in the envelope that surrounds $\mathrm{J} 18354 \mathrm{~S}$. This is in good agreement with gas temperatures derived from CO spectral line fitting $(18-20 \mathrm{~K}$; Carolan et al. 2009). The envelope appears to be slightly warmer 

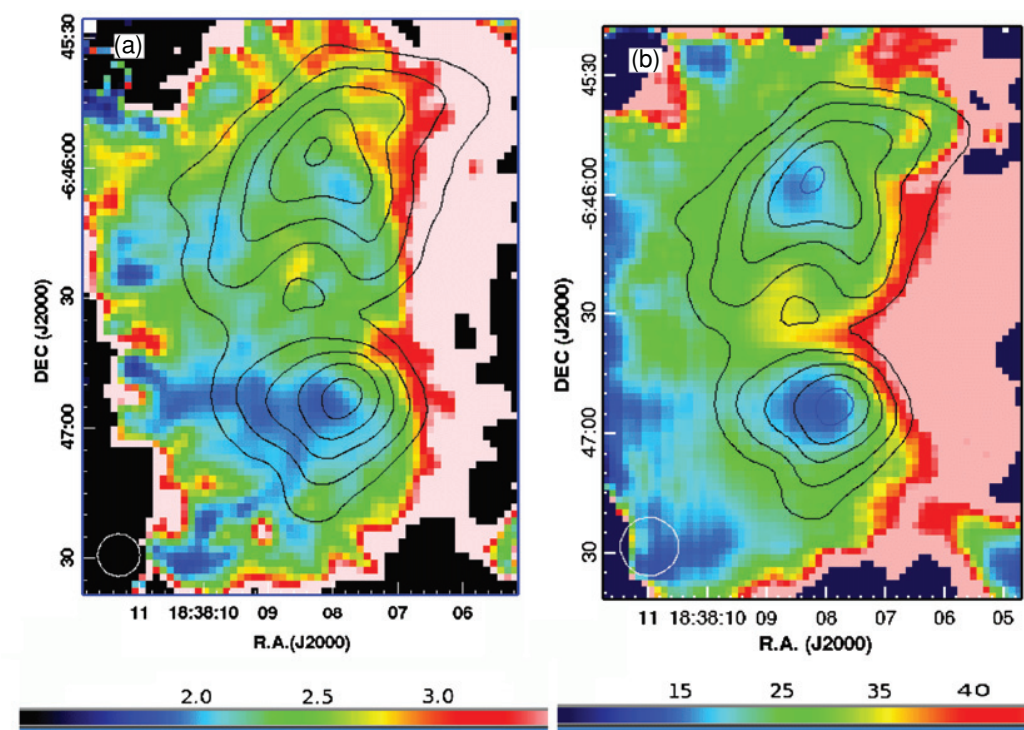

Figure 2. Color maps showing flux density ratios for (a) $350 \mu \mathrm{m} / 450 \mu \mathrm{m}$ and (b) $350 \mu \mathrm{m} / 850 \mu \mathrm{m}$ emission. The contours are of the CSO $350 \mu \mathrm{m}$ emission (levels are the same as in Figure 1). The circles in the lower-left corner of each panel indicate the resolution of the ratio map [9".4 in (a) and 15" in (b)].
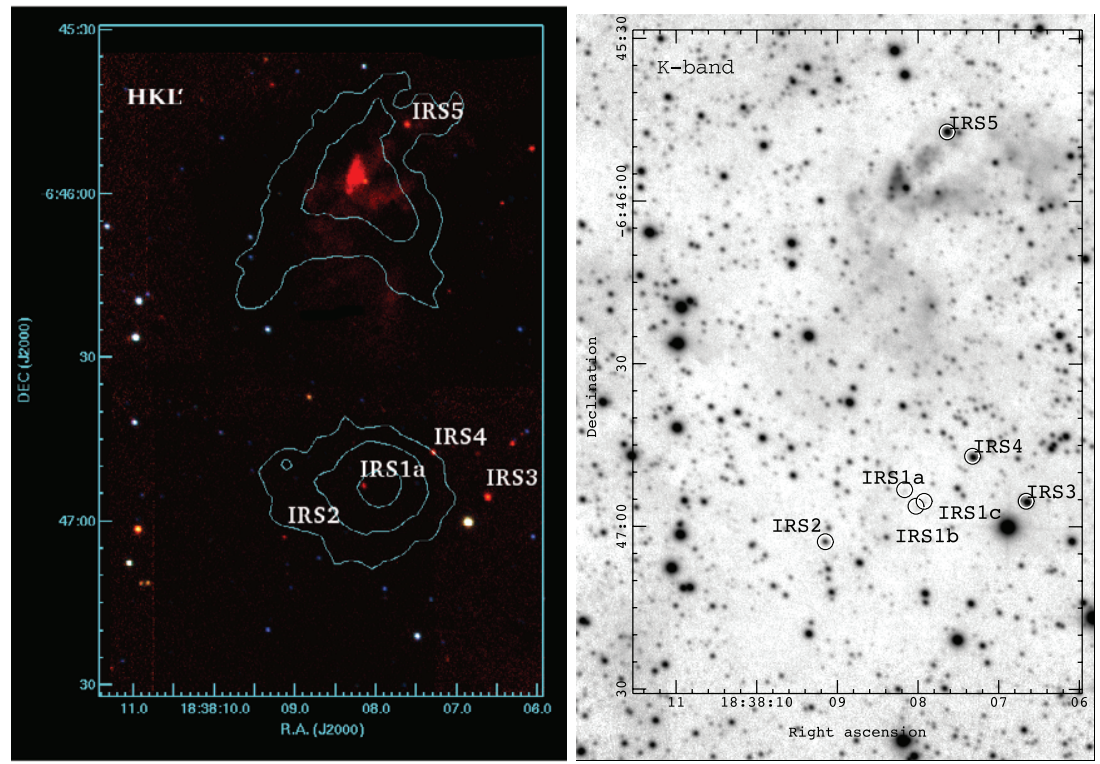

Figure 3. Left: a three-color $H K L^{\prime}$-band image with $L^{\prime}$ coded in red, $K$ in green, and $H$ in blue. Contours of the $450 \mu \mathrm{m}$ dust continuum emission are superimposed. The $K$-band image is shown to the right (gray scale with a logarithmic stretch). IRS1a, $1 \mathrm{~b}$, and $1 \mathrm{c}$ are shown more clearly in Figure 5.

than the core. Although the uncertainties in $T_{\mathrm{d}}$ and $\beta$ are large, the relatively low flux ratios of $350 / 850$ and $350 / 450$ in the cores (Figure 2) clearly point to a lower dust temperature in the center (assuming a constant $\beta$ ). This suggests that the core is heated externally, presumably by the radiation field from the $\mathrm{H}$ in region, G25.4NW. Jorgensen et al. (2006) discuss the effect a strong interstellar radiation field (ISRF) has on protostellar envelopes in Orion; they show that the dust temperature can increase in the outer regions ( $\left.r \gtrsim 10^{4} \mathrm{AU}\right)$ of the envelope due to external heating when the ISRF is 10 times higher than the standard value. For J18354S, an angular distance of 7".5 from the core center corresponds to a distance of $0.21 \mathrm{pc}$, or $4 \times 10^{4} \mathrm{AU}$. Thus, it is possible that the dust outside the $15^{\prime \prime}$ beam of our $850 \mu \mathrm{m}$ observations is externally heated, presumably by $\mathrm{G} 25.4 \mathrm{NW}$ (this also implies that $\mathrm{J} 18354 \mathrm{~S}$ is at the same radial distance as G25.4NW; see the discussion in Section 4.2). Moreover, the recently published SMA map of the dust emission at $1.3 \mathrm{~mm}$ shows that IRS1a is located at the edge of the major dust clump MM1 (see Figure 1 in Liu et al. 2011). It is possible that a star is forming in the outer part of the core and heating up the gas in the surrounding envelope, but the majority of the dust is still cold.

Having determined $\beta$ and $T_{\mathrm{d}}$, and adopting a near distance of $5.7 \mathrm{kpc}$ (see the discussion in Section 4.2), we can now estimate the bolometric luminosity, $L_{\mathrm{bol}}$, of $\mathrm{J} 18354 \mathrm{~S}$ by integrating over the spectral energy distribution (SED) of this object. Based on the fluxes listed in Table 2, for a $15^{\prime \prime}$ aperture, we estimate that $L_{\text {bol }} \sim 6.7 \times 10^{3} L_{\odot}$. If we assume that all the stellar photons are absorbed by the dust and re-emit in far-IR and submillimeter wavelengths, we can then set an upper limit to the central source's luminosity, $L_{*}$, of $\sim 6.7 \times 10^{3} L_{\odot}$. Such a value corresponds to the luminosity of a B star.

The total dust/gas mass of J18354S can be calculated by using $M=S_{v} D^{2} / \kappa_{\nu} B_{v}\left(T_{\mathrm{d}}\right)$, where $S_{v}$ is the flux at frequency 
Table 3

Near-IR Photometric Data for the Red Stars Associated with the Two Cores in JCMT 18354-0649

\begin{tabular}{|c|c|c|c|c|c|}
\hline Name & R.A. (J2000) & Decl. (J2000) & $\begin{array}{c}H \\
(\mathrm{mag})^{\mathrm{a}}\end{array}$ & $\begin{array}{c}K \\
(\mathrm{mag})^{\mathrm{a}}\end{array}$ & $\begin{array}{c}L^{\prime} \\
(\mathrm{mag})^{\mathrm{a}}\end{array}$ \\
\hline IRS1a & 183808.167 & -64653.19 & $>20.8$ & $17.9(0.1)$ & $11.20(0.1)$ \\
\hline IRS1b & 183808.026 & -64656.24 & $>20.8$ & $18.3(0.1)$ & . \\
\hline IRS1c & 183807.925 & -64655.34 & $19.1(0.1)$ & $18.1(0.1)$ & \\
\hline IRS2 & 183809.15 & -64702.8 & $18.0(0.1)$ & $14.83(0.1)$ & $12.24(0.1)$ \\
\hline IRS3 & 183806.66 & -64655.3 & $17.4(0.2)$ & $12.98(0.2)$ & $9.97(0.1)$ \\
\hline IRS4 & 183807.325 & -64647.02 & $17.9(0.4)$ & $13.7(0.2)$ & $10.73(0.1)$ \\
\hline IRS5 & 183807.64 & -64547.2 & $16.1(0.1)$ & $12.96(0.1)$ & $10.45(0.1)$ \\
\hline
\end{tabular}

Note. ${ }^{a}$ Measured near-IR magnitudes from the UKIRT data; errors are in parentheses. Zero magnitudes corresponds to 1040,645 , and $249 \mathrm{Jy}$ in $H, K$, and $L^{\prime}$, respectively.

$v, D$ is the distance, $B_{v}\left(T_{\mathrm{d}}\right)$ is the Planck function, and $\kappa_{v}$ is the dust opacity per unit gas mass. Using a dust temperature $T_{\mathrm{d}}=18 \mathrm{~K}$, a dust to gas ratio of 0.01 , and $\kappa_{850}=0.024 \mathrm{~cm}^{2} \mathrm{~g}^{-1}$ which is calculated based on $\beta=2.3$ and with the reference value $\kappa(1300 \mu \mathrm{m}) \sim 0.9 \mathrm{~cm}^{2} \mathrm{~g}^{-1}$ (for dust grains with thin ice mantles; see Ossenkopf \& Henning 1994), we derived a total mass of $M=670 M_{\odot}$ within a $15^{\prime \prime}$ aperture. For comparison, the northern core has $450 M_{\odot}$ within the same aperture, based on $\beta=2$ and $T_{\mathrm{d}}=24.5 \mathrm{~K}$. The southern core appears to be a more compact, massive dust core and has the potential to form more massive stars or clusters. The core masses and sizes are similar to massive protostars seen toward other star-forming regions (Brand et al. 2001). However, given the uncertainties in $\beta$ and $T_{\mathrm{d}}$, we estimate the masses to be accurate within a factor three.

\subsection{Embedded Young Stars in and around J18354S}

From Figure 1 we can see that the $8 \mu \mathrm{m}$ emission is dominated by the northern core. The emission in this band is mostly from hot dust PAHs heated by young stars. The northern core is typical of $\mathrm{H}$ II regions, which are full of PAH emission (e.g., Phillips $\&$ Ramos-Larios 2008). Because PAH emission is present in the extended region illuminated by the radiation, it can be used to trace the UV radiation field (Berné et al. 2009). In stark contrast, the southern core exhibits very little PAH emission. This indicates that there are no massive stars acting as heating sources, or that the stars are still heavily embedded.

At $8 \mu \mathrm{m}$ a point-like source is clearly detected near the center of the southern submillimeter core (Figure 1). This "point source" is resolved in our UKIRT images, which have a higher spatial resolution $\left(\sim 0^{\prime} .6\right)$. Figure 3 presents a three-color $H K L^{\prime}$ band image with $L^{\prime}$ in red, $K$ in green, and $H$ in blue, along with contours of the $450 \mu \mathrm{m}$ dust continuum emission for reference. We also show a deep $K$-band image on which we label the reddest of the resolved $K$-band point sources. The depth of the three-color image is limited by the $L^{\prime}$-band data which have a lower sensitivity compared to the $H$ and $K$ bands. Thus, the stars in this image include only those detected in the $L^{\prime}$ band; many more background stars and weak sources show up in the $H$ - and $K$-band observations. The red stars in Figure 3 are potentially the most interesting sources, since they are candidates for embedded protostars. Table 3 lists photometry data for the red stars near the SCUBA core, all of which have $H-K>3$ and $K-L^{\prime}>2.5$, colors that are typical of embedded protostars (Aspin \& Sandell 1997; Meyer et al. 1997).

Near the center of J18354S there is a very red star which we label IRS1a. In fact, it is the reddest star in the whole region, with $H-K>2.1$ and $K-L^{\prime}=6.7$. The $K-L^{\prime}$ color in particular is larger than that of most low-mass protostars and high-mass young stellar objects (YSOs; e.g., Aspin \& Sandell 1997; Maercker et al. 2006). The source is clearly detected in the $L^{\prime}$ band with a magnitude of 11.2 , but is relatively weak in the $K$ and $H$ bands ( $K=17.9$ and $H>20.0$ ). The extremely red colors of IRS1a could be due to high extinction; a B-type star has intrinsic colors of $H-K \sim-0.04$ and $K-L^{\prime} \sim-0.06$ (Tokunaga 2000). An extinction of $A_{\mathrm{v}}=50$ (not unreasonable for a source at low galactic latitudes and within the first galactic quadrant; $l \sim 25.4, b \sim-0.10$ ) due to intervening clouds of gas and dust would result in reddening to colors of $H-K \sim 4.0$ and $K-L^{\prime} \sim 5.4$ (for a reddening law $R_{\mathrm{v}}=5.0$; Cardelli et al. 1989). However, these colors could also be due to IR excess associated with a dusty disk and gas envelope. Without an $H$-band detection, it is difficult to distinguish between these two reddening mechanisms with the NIR data from UKIRT alone. In the following we will combine the NIR data with MIR data from Spitzer to rule out the possibility that it is just a reddened source foreground to the core. Furthermore, in Section 3.5 we will use YSO models to fit the SED of this source in order to estimate the mass of this highly reddened object.

The Spitzer data from the GLIMPSE survey confirm the existence of the reddened source IRS1a. It was identified as an Extended Green Object (EGO) in the catalog of EGOs in the GLIMPSE survey (Cyganowski et al. 2008). However, only the $4.5 \mu \mathrm{m}$ flux densities were listed for this source in the EGO catalog. Here we present more detailed analysis of the Spitzer data. In Figure 1, IRS1a is evident at $8.0 \mu \mathrm{m}$ as a point source $5^{\prime \prime}-10^{\prime \prime}$ east of the $350 \mu \mathrm{m}$ dust peak in J18354S. In Figure 4 we present a three-color image of the entire region constructed from IRAC data, with the $3.6 \mu \mathrm{m}, 4.5 \mu \mathrm{m}$, and $8.0 \mu \mathrm{m}$ bands (hereafter [3.6], [4.5], and [8.0]) color-coded blue, green, and red, respectively. At the position of IRS1a there is a greenishcolored source which indicates excess emission at $4.5 \mu \mathrm{m}$. This is very prominent, even in such an area with two massive starforming $\mathrm{H}$ II regions. The $\mathrm{HII}$ region toward the lower left in this figure is associated with W42, which is a foreground source judging from its radial velocity of $65 \mathrm{~km} \mathrm{~s}^{-1}$ (compared with $96 \mathrm{~km} \mathrm{~s}^{-1}$ for J18354S). The green feature associated with IRS1a is detected in all four IRAC bands (although it is not prominent at $3.5 \mu \mathrm{m}$ and $8.0 \mu \mathrm{m}$ ), and it appears to be extended. The extension is most prominent at $4.5 \mu \mathrm{m}$, so it seems likely that the emission is due to shock-excited spectral lines, e.g., the $\mathrm{H}_{2}$ 0-0 S(9) line at $4.694 \mu \mathrm{m}$, or the $\mathrm{CO}$ fundamental band head at 4.5-4.9 $\mu \mathrm{m}$. Note that pure-rotational $\mathrm{H}_{2}$ emission has been detected in a number of low- and high-mass star-forming regions (e.g., Davis et al. 2007, 2008; Dionatos et al. 2009; Neufeld et al. 2009). A two-dimensional Gaussian fit to the feature at $4.5 \mu \mathrm{m}$ yields an FWHM size of $5^{\prime \prime} \times 7^{\prime \prime} .5$ orientated at a position angle of $37 \mathrm{deg}$. If this feature represents a molecular hydrogen jet, then at a distance of $5.7 \mathrm{kpc}$ the major axis of the Gaussian fit corresponds to a physical jet length of $0.2 \mathrm{pc}$.

To search further for shock-excited $\mathrm{H}_{2}$ emission, we have used the UKIRT imager UIST with a narrowband filter centered on the $\mathrm{H}_{2} 1-0 \mathrm{~S}(1)$ rotational-vibrational line at $2.12 \mu \mathrm{m}$. However, we did not detect any extended line emission in the limited observing time available to us (10 minutes of integration). Although the 1-0 $\mathrm{S}(1)$ line requires gas heated to higher temperatures $(T \sim 2000 \mathrm{~K})$ than the pure-rotational transitions seen at longer wavelengths with Spitzer $(T \sim 700 \mathrm{~K})$, both transitions are generally observed in molecular outflows from young stars (Davis et al. 2007; Dionatos et al. 2009; Neufeld 


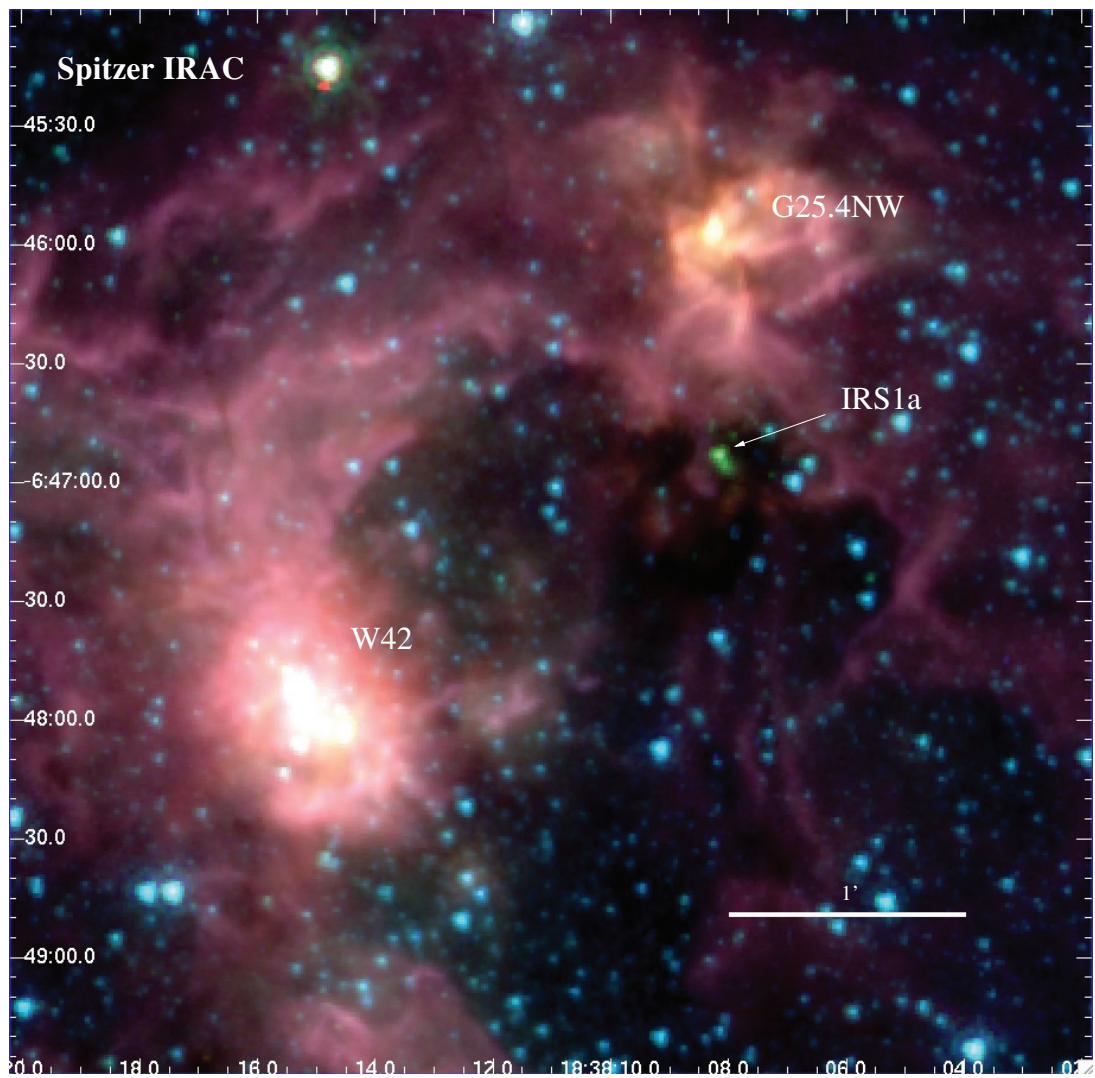

Figure 4. Spitzer IRAC three-color image ( $3.6 \mu \mathrm{m}$ in blue, $4.5 \mu \mathrm{m}$ in green, and $8.0 \mu \mathrm{m}$ in red) of the entire IRAS 18354-0649 region. The white bar in the figure measures 1 arcmin.

et al. 2009), particularly since bow-shaped shocks are known to produce a range of excitation conditions (e.g., Smith 1991). It seems likely, therefore, that our non-detection at $2.12 \mu \mathrm{m}$ is due to observations that are too shallow, and to the high extinction toward this embedded source. $\mathrm{CO}$ band head emission requires high densities for excitation and is unlikely to be seen in extended regions, though excited $\mathrm{CO}$ may also contribute to the observed flux.

\subsection{Source Multiplicity in the J18354S Core}

Rather than a jet, the extended green feature in the Spitzer image in Figure 4 could simply represent two or more unresolved point sources. To explore this probability, in Figure 5 we overlay the $4.5 \mu \mathrm{m}$ contours associated with the green feature on to our high-resolution $K$-band image. The latter indicates the presence of three very faint sources within the central $5^{\prime \prime}$ of the SCUBA core: IRS1a, but also IRS1b and IRS1c. Of these, IRS1c appears strongest in the $H$-band image, but is undetected in the UKIRT $L^{\prime}$-band data and in the IRAC $3.6 \mu \mathrm{m}$ band image. It has a relatively shallow color index, $H-K=1$ (Table 3 ), so it is probably a reddened field star rather than an embedded protostar with an infrared excess. IRS1b, like IRS1a, is undetected in $H$, though (like IRS1a) it appears to have a very red NIR color and is certainly a candidate YSO. Note, however, that it is undetected in the $L^{\prime}$-band image and is barely detected in IRAC bands 1 to 3 (it is undetected at $8.0 \mu \mathrm{m}$ ).

Because of the extended emission, it is difficult to accurately measure the IRAC fluxes. As a very rough estimate, we ignore the extended emission and assume that all the fluxes come from IRS1a and 1b. We used an aperture of four native pixels (4".8) centered on each source. These two apertures overlap with one

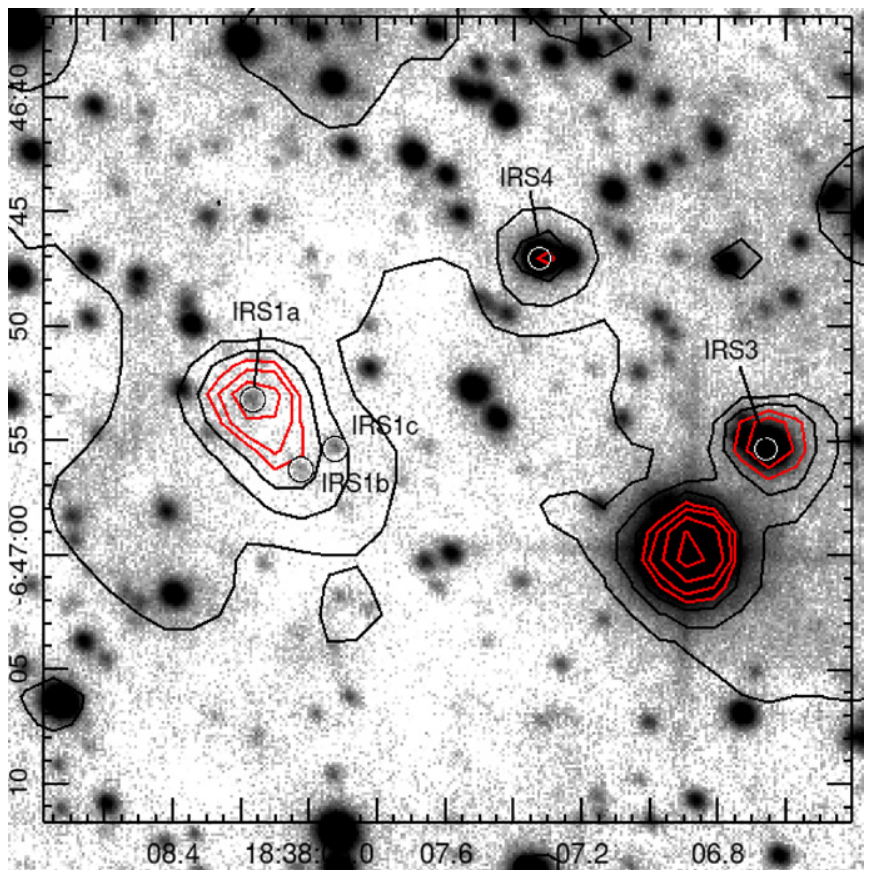

Figure 5. $K$-band image of $\mathrm{J} 18354 \mathrm{~S}$ with the near-IR sources labeled and Spitzer IRAC $4.5 \mu \mathrm{m}$ contours overlaid. The peak of the extended feature at $4.5 \mu \mathrm{m}$ (seen in green in Figure 4) clearly coincides precisely with IRS1a. The extension to the southwest may be associated with IRS1b and IRS1c.

pixel, and we assigned half of the pixel flux to each source. The IRAC [3.6], [4.5], and [5.6] mag of IRS1b are measured as $12.6 \pm 0.2,10.3 \pm 0.2$, and $9.0 \pm 0.2$, respectively. For the 
Table 4

Near- and Mid-IR Photometry for IRS1a, with Submillimeter Fluxes for the Associated Core J18354S

\begin{tabular}{lccccc}
\hline \hline Instrument & $\begin{array}{c}\lambda \\
(\mu \mathrm{m})\end{array}$ & $\mathrm{Mag}^{\mathrm{a}}$ & $\begin{array}{c}S^{\mathrm{b}} \\
(\mathrm{mJy})\end{array}$ & $\begin{array}{c}\text { Error } \\
(\%)\end{array}$ & $\begin{array}{c}\text { Aperture }^{\mathrm{c}} \\
(\operatorname{arcsec})\end{array}$ \\
\hline UIST $H$ & 1.64 & $>20.8$ & 0.01 & 10 & 2 \\
UIST $K$ & 2.20 & 17.9 & 0.045 & 10 & 2 \\
UIST $L^{\prime}$ & 3.77 & 11.2 & 8.2 & 10 & 2 \\
IRAC 1 & 3.6 & 11.3 & 8.8 & 20 & 5 \\
IRAC 2 & 4.5 & 9.2 & 39 & 20 & 5 \\
IRAC 3 & 5.8 & 7.8 & 89 & 25 & 5 \\
IRAC 4 & 8.0 & 7.2 & 83 & 25 & 5 \\
MIPS & 24 & $\ldots$ & $<760$ & $\mathrm{n} / \mathrm{a}$ & 10 \\
MIPS & 70 & $\ldots$ & $<110000$ & $\mathrm{n} / \mathrm{a}$ & 20 \\
SHARC II & 350 & $\ldots$ & 48000 & 25 & 9.4 \\
SCUBA & 450 & $\ldots$ & 19000 & 20 & 8.0 \\
SCUBA & 850 & $\ldots$ & 4500 & 15 & 15 \\
\hline
\end{tabular}

Notes.

${ }^{a}$ Measured magnitudes from UKIRT and Spitzer.

b Measured fluxes in mJy; note that zero magnitudes correspond to 1040, 645, and $249 \mathrm{Jy}$ in $H, K$, and $L^{\prime}$, respectively.

c The 350, 450, and $850 \mu \mathrm{m}$ values are measured in a 9.'4, 8", and $15^{\prime \prime}$ beam, while the near-IR data use a $2^{\prime \prime}$ aperture. Our sub-arcsecond resolution $H$-, $K$-, and $L^{\prime}$-band images represent the highest resolution observations of this region ( $1^{\prime \prime}$ corresponding to $0.028 \mathrm{pc}[5700 \mathrm{AU}]$ at a distance of $5.7 \mathrm{kpc}$ ).

non-detection band at $8.0 \mu \mathrm{m}$, we estimate an upper limit of 7.9 mag. Comparing with the colors of jets and shocked gas in the IRAC [3.6] - [4.5] versus [4.5] - [5.8] diagram of Ybarra \& Lada (2009; Figure 1), we find that IRS1b has a color similar to that of Class $0 /$ I objects rather than a pure jet. Thus, IRS $1 \mathrm{~b}$ could be an embedded protostar similar to IRS1a. The extended taillike feature may be the partially resolved counterpart of IRS1b, judging from its IRAC color. However, we cannot completely rule out the existence of shocked gas surrounding the protostars, as outflows are clearly seen on the $\mathrm{HCN}(3-2)$ map from the SMA (Liu et al. 2011). If part of the extended emissions are indeed from shocked gas, the measured fluxes at $4.5 \mu \mathrm{m}$ (and possibly at $5.8 \mu \mathrm{m}$ ) should be used as upper limits.

Table 4 lists photometry for IRS1a in the $H, K$, and $L^{\prime}$ bands and at the four IRAC wavelengths. These values show that IRS1a has mid-IR colors, [3.6] $-[4.5]=2.1$ and [5.8] $-[8.0]=0.6$, that are similar to Class 0 and Class I protostars, though also to more evolved YSOs (note that inclination effects have a considerable effect on these colors; Allen et al. 2004). The midIR photometry in Table 4 includes emission from the extended green feature in Figure 4; we note that the [3.6] - [4.5] color is consistent with sources which are known to drive molecular outflows (which are typically redder in [3.6] - [4.5] than most YSOs; Davis et al. 2008) and with the predictions of fast Ctype bow shocks or J-shocks (Davis et al. 2008; Ybarra \& Lada 2009).

Using the [3.6] - [4.5] versus [5.8] - [8] diagram of Figure 23 in Robitaille et al. (2006), we found that both IRS1a and 1b have the color of stage I sources. If IRS1a was a stage II source, the visual extinction would be $A_{\mathrm{v}} \sim 85$, which means that the $K$-band magnitude is 6.8 and thus has a absolute magnitude of -7 (at a distance of $5.7 \mathrm{kpc}$ ), equivalent to an O-type star which should generate a bolometric luminosity of $\sim 6 \times 10^{5} L_{\odot}$, much higher than what we measured. Similarly, if IRS1b were a reddened star or stage II source, the high [3.6] - [4.5] value indicates an extremely high visual extinction $A_{\mathrm{v}} \sim 100$, and therefore an absolute $K$-band magnitude of -14 (O-type star), with unrealistically high luminosity.

Although IRS1a is 3-4 times more luminous than IRS1b in the IRAC bands, we cannot conclude that it is more massive than IRS1b because the MIR fluxes of YSOs are dominated by disks rather than the stellar photospheres, and they depend on disk orientation and evolutionary stage. More data are needed to determine the SED and mass of IRS1a and 1b. Nevertheless, the high-resolution interferometric maps at the $\mathrm{HCN}(3-2)$ transition clearly show that IRS $1 \mathrm{a}$ is located at the center of the blueshifted and redshifted lobes of the outflow contours (see Figure 7 of Liu et al. 2011), and most of the dust and $\mathrm{CH}_{3} \mathrm{OH}$ emission are associated with IRS1a rather than IRS1b (see Figures 1 and 2 of Liu et al. 2011). Thus, we assume that IRS1a is the major source heating the nearby dust and driving the outflow in J18354S. Note that IRS1a and IRS1b are separated by $4^{\prime \prime}$, which corresponds to $0.11 \mathrm{pc}$ at a presumed distance of $5.7 \mathrm{kpc}$.

It is therefore possible that there are at least two stars inside the submillimeter core $\mathrm{J} 18354 \mathrm{~S}$, or even a cluster in the very early stages of formation. High-resolution interferometric maps reveal three dust clumps in J18354S (Liu et al. 2011), suggesting that fragmentation is taking place in this core. Deep, highspatial resolution MIR images may reveal the embedded stellar population (the James Webb Space Telescope will be ideal for such a project).

\subsection{Mid-IR and Far-IR Emission}

Figures 6(a) and (b) show MIPS $24 \mu \mathrm{m}$ and $70 \mu \mathrm{m}$ images of the same region as in Figure 4. Contours of the SCUBA $450 \mu \mathrm{m}$ emission are overlaid, showing the location of the two SCUBA cores $\mathrm{J} 18354 \mathrm{~N}$ and J18354S. We can see that IRS1a is located in a well illuminated area; the two $\mathrm{H}$ II regions are evident as saturated nebulae in these images. IRS1a itself is bathed in diffuse background emission. Consequently, we were unable to extract long-wavelength MIR photometry from the Spitzer data, although upper limits to the $24 \mu \mathrm{m}$ and $70 \mu \mathrm{m}$ fluxes are listed in Table $4 .{ }^{9}$ Note that at $70 \mu \mathrm{m}$ the instrumental pointspread function is quite broad, and therefore the source would have to be quite bright to stand out. The $24 \mu \mathrm{m}$ and $70 \mu \mathrm{m}$ upper limits in Table 4 indicate that the emission from IRS1a is relatively weak (certainly in comparison to the $\mathrm{H}$ II regions nearby). This suggests that the protostar is still very young and that the dust is relatively cold.

\subsection{SED Modeling}

The fluxes from near-IR to submillimeter wavelengths for $\mathrm{J} 18354 \mathrm{~S}$ are listed in Table 4. We use the peak fluxes from the central beam for the submillimeter data points because the source is compact and is less than $12^{\prime \prime}$ in diameter. The beam size at $850 \mu \mathrm{m}$ is much larger than that at 350 and $450 \mu \mathrm{m}$. As discussed in Section 3.1, part of the dust in the envelope could be externally heated; thus we assume $50 \%$ of the $850 \mu$ m fluxes in the $15^{\prime \prime}$ beam are from the central YSO and use this value (2.15 Jy) for our SED fitting.

The SEDs associated with IRS $1 \mathrm{a}$ and the J18354S core overall were analyzed by fitting model SEDs to each observed source from a large pre-computed grid of YSO models (Robitaille et al.

\footnotetext{
9 To obtain the $24 \mu \mathrm{m}$ and $70 \mu \mathrm{m}$ upper limits for IRS1a we subtracted the background flux and added $2 \sigma$ (with $\sigma$ equal to the rms) as an initial estimate; we then subtract the source with the flux of the estimated upper limit. One should see a slight oversubtraction if the upper limit is sensible. We repeated this exercise to arrive at our best estimates of the upper limit in each band.
} 

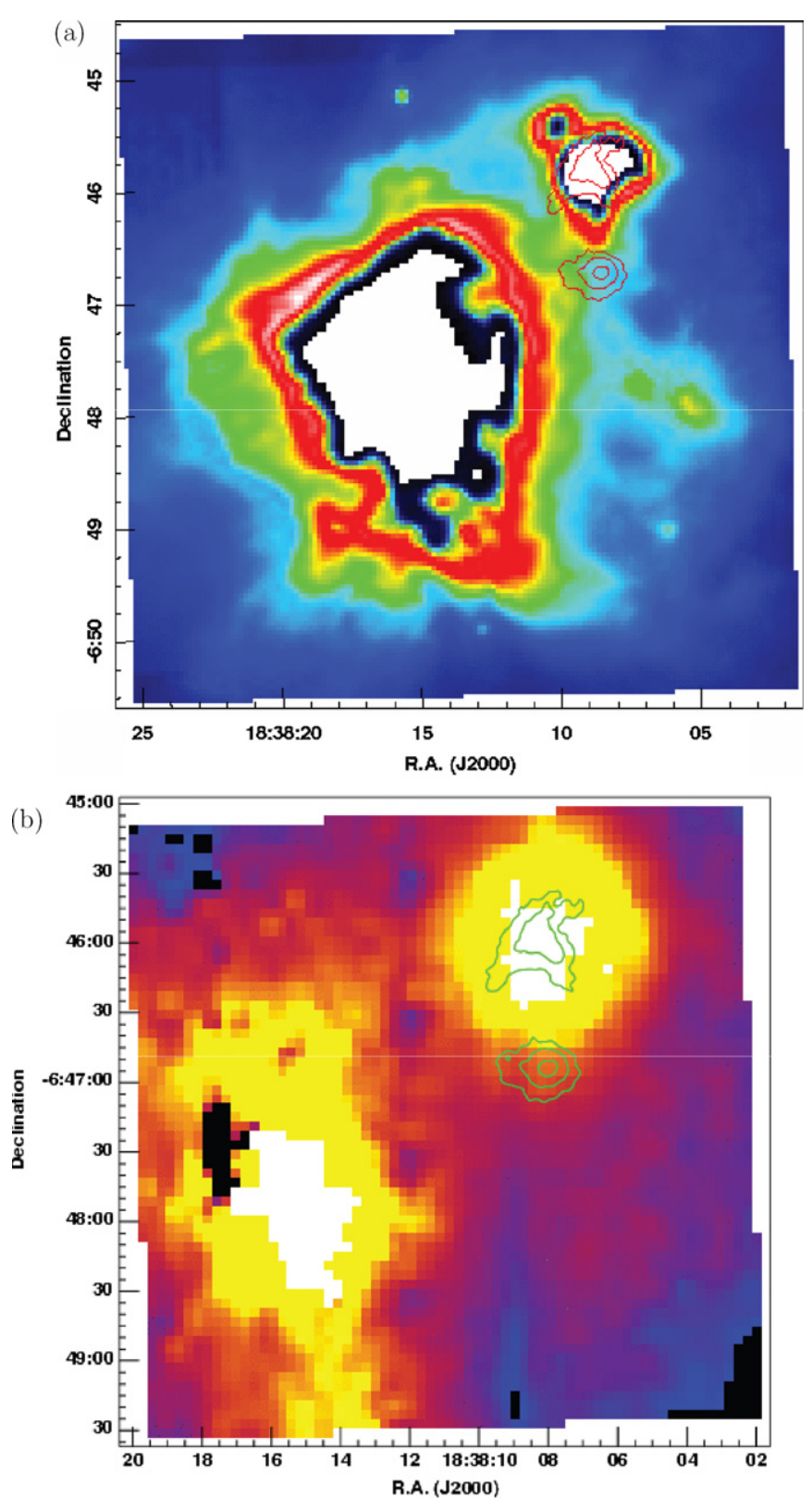

Figure 6. Spitzer MIPS images at (a) $24 \mu \mathrm{m}$ (top) and (b) $70 \mu \mathrm{m}$ (bottom) of the region shown in Figure 4. SCUBA $450 \mu \mathrm{m}$ contours, showing the locations of J18354N and J18354S, are overlaid for comparison.

2006, 2007). The grid consists of 20,000 two-dimensional YSO radiation transfer models spanning a complete range of stellar mass and evolutionary stage. Each YSO model outputs SEDs at 10 viewing angles (inclinations), so the fitter actually has 200,000 SEDs to choose from. The YSO models also output SEDs for each of 50 aperture sizes. Thus, if the distance to the star formation region is known, only the model SEDs with the appropriate aperture size compared to the observed photometry are used.

For the purposes of the fitting, upper limits were used for MIR $24 \mu \mathrm{m}$ and $70 \mu \mathrm{m}$ data as well as for the $4.5 \mu \mathrm{m}$ IRAC data because the source appears to have excess flux in this bandpass due to molecular emission associated with outflow activity. The fitting procedure is similar to that of Shepherd et al. (2007). Figure 7(a) shows the best-fit models and Table 5 (line 1) presents the best-fit model parameters for IRS1a, which
Table 5

SED Fitting Parameters ${ }^{\mathrm{a}}$ for the Three Models Shown in Figure 7

\begin{tabular}{lcrrrcc}
\hline \hline Model $^{\text {b }}$ & Incl. & $\chi^{2}$ & $\begin{array}{c}A_{\mathrm{v}} \\
(\mathrm{mag})\end{array}$ & $\begin{array}{c}M_{*} \\
\left(M_{\odot}\right)\end{array}$ & $\begin{array}{c}L_{\text {tot }} \\
\left(L_{\odot}\right)\end{array}$ & $\begin{array}{c}\dot{M}_{\text {env }} \\
\left(M_{\odot} \mathrm{yr}^{-1}\right)\end{array}$ \\
\hline (a) & 18.2 & 269.6 & 34.7 & 11.7 & $2.86 \times 10^{3}$ & $3.8 \times 10^{-4}$ \\
(b) & 75.5 & 0.5 & 0.0 & 17.8 & $8.89 \times 10^{3}$ & $3.9 \times 10^{-4}$ \\
(c) & 87.1 & 135.1 & 17.4 & 6.4 & $1.26 \times 10^{3}$ & $1.0 \times 10^{-7}$ \\
\hline
\end{tabular}

Notes.

${ }^{a}$ The parameters are described in detail in Robitaille et al. (2006).

b The SED models shown in Figure 7: (a) fitted with all flux data points, (b) fitted with only the data points with $\lambda>50 \mu \mathrm{m}$, and (c) fitted with only the data points with $\lambda<50 \mu \mathrm{m}$.

suggest that a type B star with $11.7 M_{\odot}$ is forming inside the submillimeter core.

However, strictly speaking, no YSO model can fit all of the data points perfectly. The best fit shown in Figure 7(a) still has $\chi^{2}=269$; the major discrepancy is at the submillimeter wavelengths, particularly $350 \mu \mathrm{m}$ and $450 \mu \mathrm{m}$. All of the YSO models that can fit the NIR and MIR parts of the SED predict much less submillimeter emission, and there appears to be excess submillimeter emission that could not be explained by the energy generated by the central YSO. To further investigate this, we have also fitted the NIR/MIR data and the submillimeter data separately. Figure 7(b) shows the fit for the submillimeter data only, and the fitting parameters are listed in Table 5. The submillimeter SEDs could be well fitted with models with $M=$ 13-18 $M_{\odot}$ and a total luminosity of $5-10 \times 10^{3} L_{\odot}$, with $\chi^{2}$ less than 2 . These submillimeter fluxes are mostly blackbody emission from the envelope which reveal the total energy reprocessed by the dust. If most of the energy is generated from the central source, it requires an early (B) type star. However, the NIR part of the SED can be best fitted with YSO models with $M=6-8 M_{\odot}$ (Figure 7(c), $\chi^{2}$ less than 200). In this case the total luminosity generated by the star is $1-2 \times 10^{3} L_{\odot}$, which can account for only $30 \%$ of the luminosity seen at submillimeter wavelengths.

Possible explanations for the excess submillimeter emission are as follows.

1. Contamination from external sources, such as the northern $\mathrm{H}_{\text {II }}$ region (as is the case at $70 \mu \mathrm{m}$ ). From the SCUBA and CSO SHARC maps we can clearly see that J18354S is the dominant source of the submillimeter emission in this region; the contribution from outside heating sources should therefore be less than $20 \%-30 \%$.

2. There is more than one YSO inside the submillimeter core, resulting in excess submillimeter emission. As discussed in Section 3.3, the other candidate YSO inside the submillimeter core, IRS1b, produces at least $\sim 30 \%$ as much MIR flux as IRS1a. Consequently, IRS1b might account for part of the submillimeter luminosity.

3. Large-scale gravitational collapse as a heating source. The dust could be heated in the shocked region due to massive inflow and accretion. This scenario is similar to what is seen in a Class 0 object, where most of the energy is nonstellar. In the model of Whitney et al. (2003), the accretion luminosity is calculated with the formula

$$
L_{\text {acc }} \propto 3 G M_{*} \dot{M} / 2 R_{\text {dust }} .
$$

Here the stellar mass $M_{*}$ is used to calculate the accretion luminosity. This formula is based on accretion onto a single 

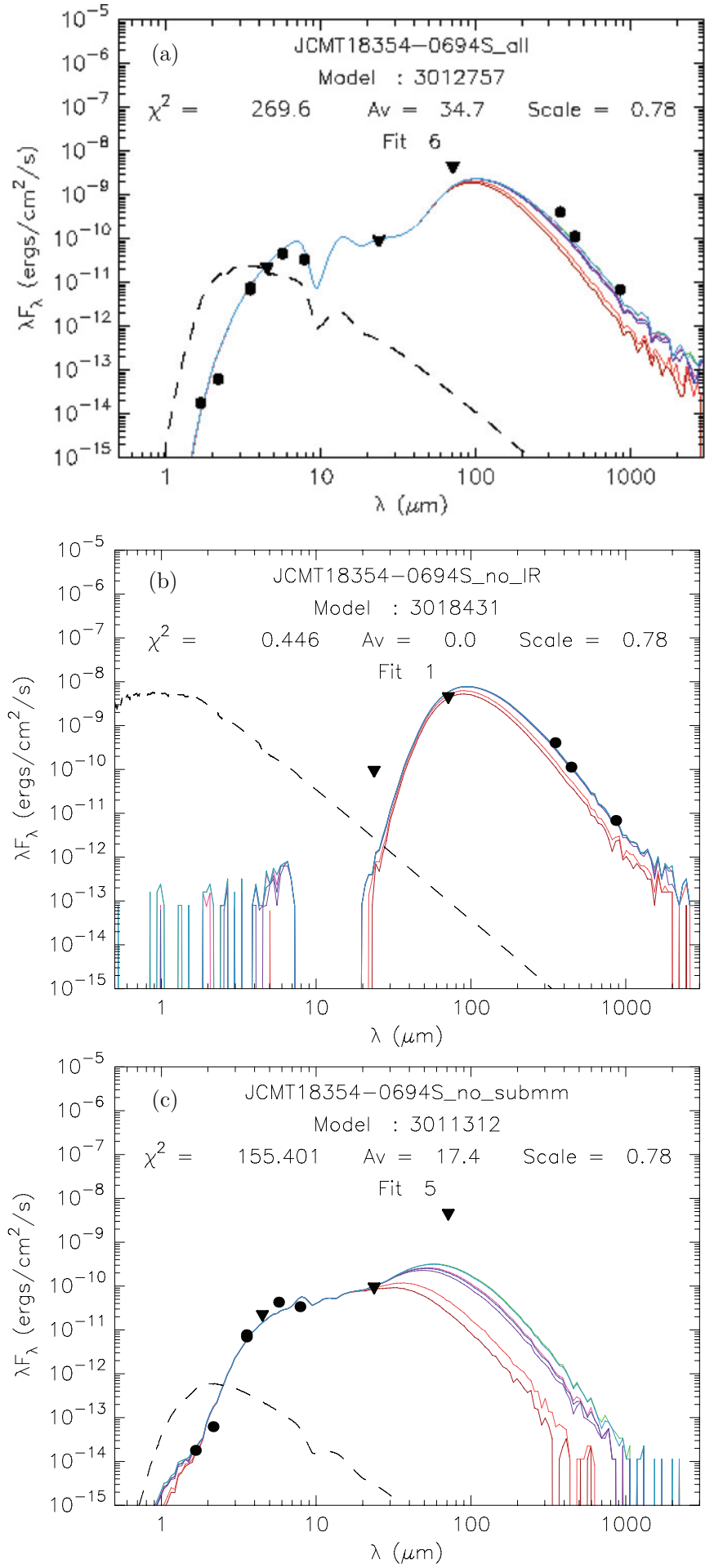

Figure 7. (a) The best YSO SED model fits for J18354S. The fitting assumes that the mid-IR flux from the millimeter core is dominated by a single source. The [4.5] upper limit (triangles) was used because the source appears to have excess flux in this bandpass due to molecular emission associated with outflow activity that has not been incorporated into the YSO models. The blue line represents the best fit to the data; red lines represent the SED observed through different apertures. The dashed line shows the photospheric flux from the best-fit YSO model as it would appear if suffering from interstellar extinction only, i.e., in the absence of circumstellar material. (b) The best-fit YSO SED model to only the submillimeter part of the SED of J18354S; the central YSO mass is $17.8 M_{\odot}$. (c) The best-fit YSO SED model to only the near-IR/mid-IR part of the J18354S spectrum; in this case the central YSO mass is $6.4 M_{\odot}$. The parameters for these three models are listed in Table 5.

(A color version of this figure is available in the online journal.) disk. However, in MSF, the inflow occurs on a much larger scale. Collapse may form a cluster rather than a single star, and the large-scale accretion would generate a luminosity larger than that of a single protostar. In fact, the infall rate from fitting the $\mathrm{HCN}(3-2)$ and $\mathrm{HCO}^{+}(3-2)$ molecular lines is $\sim 3.4 \times 10^{-3} M_{\odot} \mathrm{yr}^{-1}$ (Wu et al. 2005; Carolan et al. 2009; Liu et al. 2011), which is one order of magnitude larger than the accretion rate $\dot{M}=3.8 \times 10^{-4} M_{\odot} \mathrm{yr}^{-1}$ used in our SED modeling. Figure 1 of Liu et al. (2011) shows that several clumps are forming inside J18354S, and IRS1a is associated with only one of the clump. Thus, the energy from large-scale gravitational collapse could explain part of the excess emission seen at submillimeter wavelengths.

A similar situation is also seen in the SED fitting of other massive YSOs. For example, Figure 11 in Shepherd et al. (2007) shows that the SED model also predicts much less flux at $450 \mu \mathrm{m}$ and $850 \mu \mathrm{m}$ (see source 12 in their Table 4). Their SED model estimates a total luminosity of $6.9-9.4 \times 10^{3} L_{\odot}$, while Rathborne et al. (2005) derived a total bolometric luminosity of $32,000 L_{\odot}$ for the same source by fitting a standard graybody curve to the peak fluxes from all of their continuum data in the mid-IR $(24 \mu \mathrm{m})$, submillimeter $(350,450$, and $850 \mu \mathrm{m})$, and millimeter (1.2 mm).

For comparison, IRS1a is 1.6 times farther away, 2 times stronger at $350 \mu \mathrm{m}$ and $450 \mu \mathrm{m}$, and 5 times stronger at $5.8 \mu \mathrm{m}$ and $8.0 \mu \mathrm{m}$ than the $10 M_{\odot}$ protostar found by Rathborne et al. (2005, MM3 in their paper). It appears to be a massive protostar with a different type of SED. Future deep IR spectroscopy would be useful to obtain the spectral type of the central star and to more accurately estimate the near-IR opacity, stellar mass, and luminosity for the protostar.

\subsection{The Gas Envelope around J18354S}

$\mathrm{J} 18354 \mathrm{~S}$ is unique in that it shows signs of both inflow and outflow (Carolan et al. 2009; Wu et al. 2005). Carolan et al. modeled $\mathrm{HCN}(3-2)$ and $\mathrm{CO}(3-2)$ profiles and showed that $\mathrm{J} 18354 \mathrm{~S}$ is a scaled up version of a low-mass star-forming core in terms of kinematic structure, but with much higher supersonic internal velocities. We confirmed the existence of inflow and outflow motions in J18354S with new $\mathrm{HCN}(4-3)$ data (Figure 8), which were obtained with a higher angular resolution. Figure 8 shows a grid of spectra and contours centered at $\mathrm{J} 18354 \mathrm{~S}$. The inflow region is confined to within a (deconvolved) $\sim 10^{\prime \prime}$ radius and is coincident with IRS1a. Note that $10^{\prime \prime}$ corresponds to $0.28 \mathrm{pc}$ at a distance of $5.7 \mathrm{kpc}$. The outflow lobes appear to be slightly more extended; the region with velocities higher than the FWHM line width of $20 \mathrm{~km} \mathrm{~s}^{-1}$ is in a region of $15^{\prime \prime}$. The $\mathrm{HCN}(4-3) / \mathrm{HCN}(3-2)$ ratio is $0.78 \pm 0.15$ in the central $20^{\prime \prime}$ (after smoothing the $\mathrm{HCN}(4-3)$ data to a resolution of $\left.20^{\prime \prime}\right)$. Using the large velocity gradient (LVG) analysis code similar to that of Greve et al. (2009) for analyzing the $\mathrm{HCN}$ line ratios in Arp 220, and a procedure described in Zhu et al. (2003), we found that no LVG solution is possible with a kinetic temperature $T_{k}<10 \mathrm{~K}$ and a gas density $n<10^{6}$ $\mathrm{cm}^{-3}$, given the high $\mathrm{HCN}$ ratio measured in J18354S. However, with only one line ratio, we cannot fully constrain the physical parameters. Assuming a gas density of $1 \times 10^{6} \mathrm{~cm}^{-3}$ as estimated from the SCUBA data, we found that $T_{k}=10-20 \mathrm{~K}$, an abundance of $\left[\mathrm{HCN} / \mathrm{H}_{2}\right] \sim 2 \times 10^{-8}$ (Bergin et al. 1996; Lahuis \& van Dishoeck 2000), and a velocity gradient $d V / d R$ of 5-20 $\mathrm{km} \mathrm{s}^{-1} \mathrm{pc}^{-1}$ can yield an $\mathrm{HCN}(4-3) / \mathrm{HCN}(3-2)$ ratio of $0.65-0.85$. These parameters are consistent with the result of 

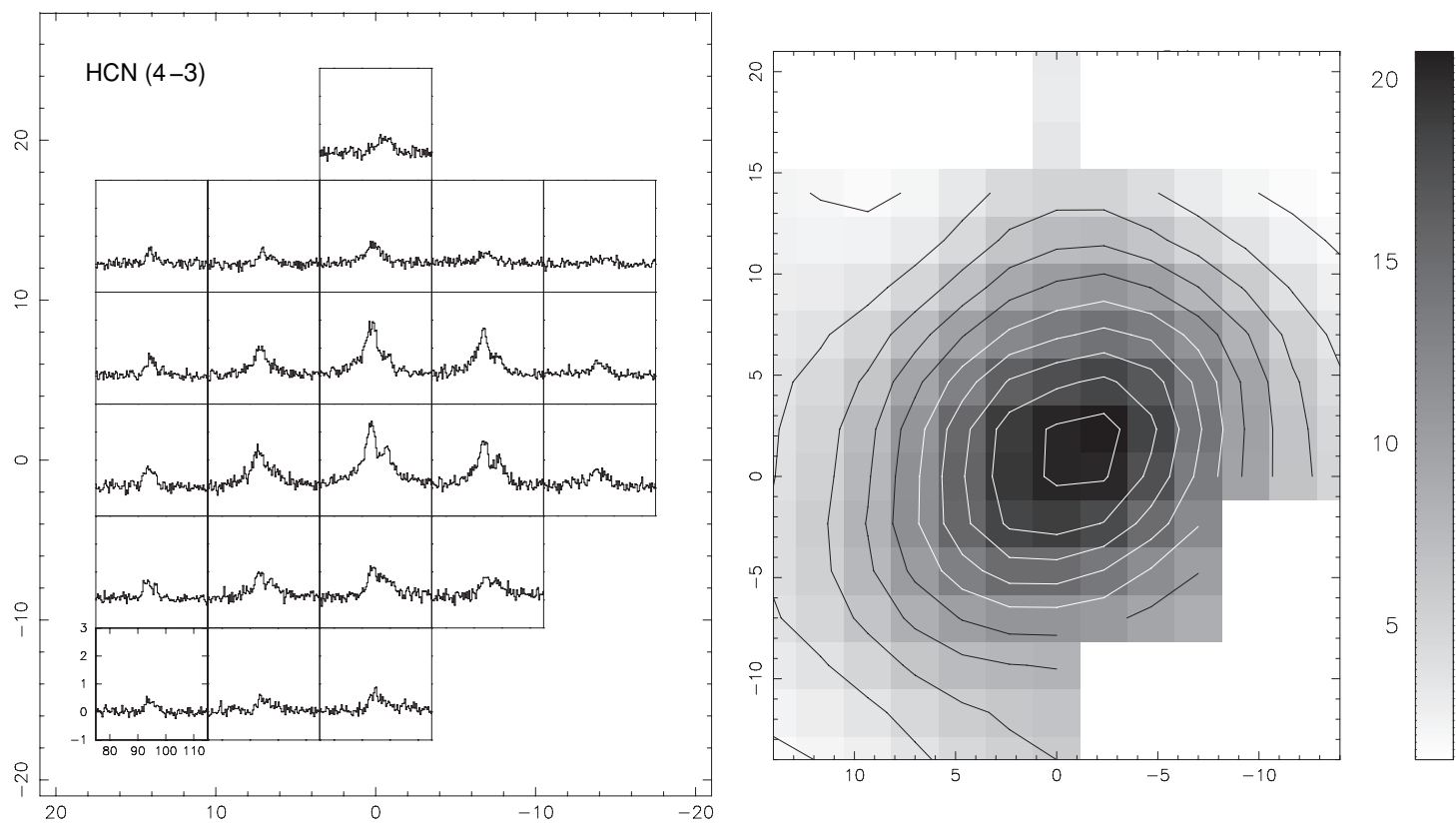

Figure 8. HCN(4-3) observations of J18354S shown as a grid of spectra (left) and as a contour plot of integrated intensity (right). The 0,0 offset position, centered on J18354S, corresponds to R.A.: 1838 08.16, decl.: -6 46 52.1 (J2000.0). Contour levels are 2.5, 5.0, 7.5, 10.0, 12.5, 15.0, 17.5, 20.0, 22.5, and 25.0 K km s${ }^{-1}$. The temperature scale is $T_{A}^{*}$ which can be converted to main-beam temperature $T_{\mathrm{mb}}$ with the formula $T_{A}^{*}=T_{\mathrm{mb}} / \eta_{\mathrm{mb}}$, where $\eta_{\mathrm{mb}}=0.63$ is the main-beam efficiency for RXB.

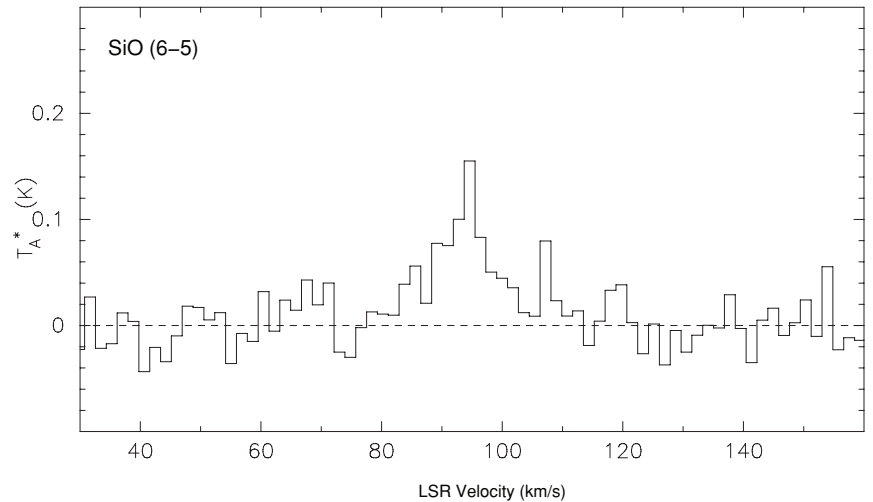

Figure 9. $\mathrm{SiO}(6-5)$ spectrum observed toward J18354S. The temperature scale is $T_{A}^{*}$ which can be converted to main-beam temperature $T_{\mathrm{mb}}$ with the formula $T_{A}^{*}=T_{\mathrm{mb}} / \eta_{\mathrm{mb}}$, where $\eta_{\mathrm{mb}}=0.69$ is the main-beam efficiency for RXA

Carolan et al. (2009) derived from fitting the $\mathrm{HCN}(3-2)$ profile. Although the line ratio may be distorted by self-absorption, it can still be used as an indicator of the large amounts of high-density gas in the J18354S core. We note also that the HCN contours appear to be extended perpendicular to the direction of the possible collimated $\mathrm{H}_{2}$ jet traced at $4.5 \mu \mathrm{m}$ in Figure 4, although high-resolution interferometric data are needed to confirm this feature.

We have also detected the $\mathrm{SiO}(6-5)$ line toward J18354S (Figure 9). The abundance of $\mathrm{SiO}$ is thought to be enhanced in regions where shocks destroy dust grains (Martin-Pintado et al. 1992; Avery \& Chiao 1996; Gibb et al. 2004). SiO emission is therefore a reliable tracer of outflow activity. The strong $\mathrm{SiO}$ line detected toward J18354S, combined with the broad line profiles of high-density molecular tracers HCN (4-3), provides further evidence that active star formation is occurring in J18354S. Moreover, Gibb et al. (2004), based on a $\mathrm{SiO}(5-4)$ survey of

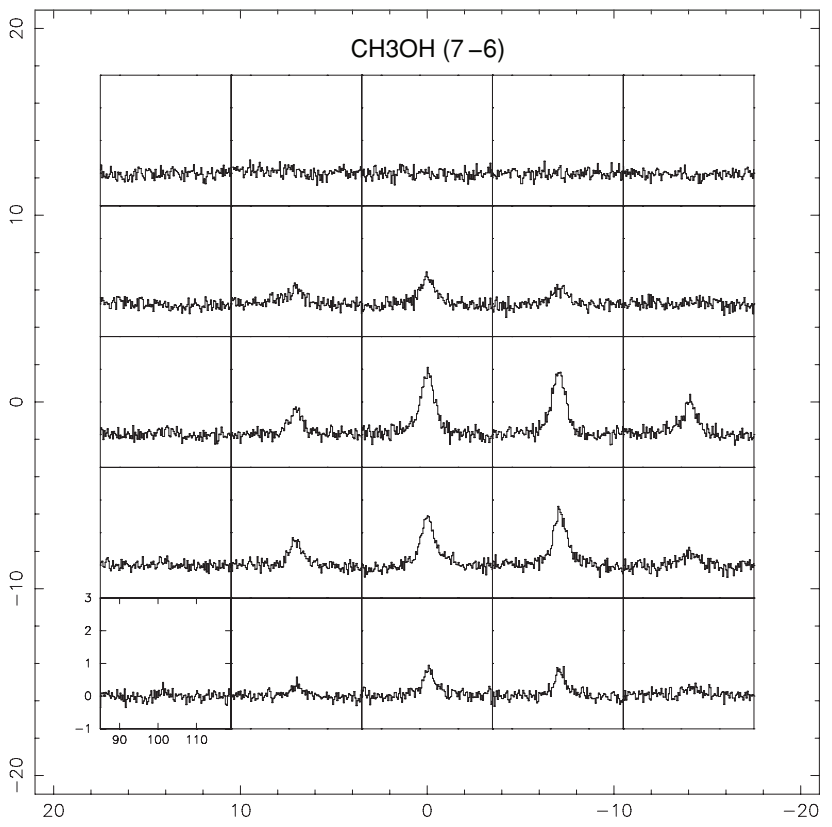

Figure 10. Grid of $\mathrm{CH}_{3} \mathrm{OH}-\mathrm{A}\left(7_{00}-6_{00}\right)$ spectra centered on J18354S (R.A.: 18 38 08.16, decl.: -64652.1 , J2000.0). The temperature scale is $T_{A}^{*}$.

outflows from low-mass protostars, suggest that $\mathrm{SiO}$ emission can be used as a signpost of outflows from the very youngest, Class 0, sources. Notably, we did not detect the $\mathrm{SiO}(6-5)$ line in the more evolved G25.4NW H II region with a similar amount of integration time.

Figure 10 shows a grid of $\mathrm{CH}_{3} \mathrm{OH}-\mathrm{A}\left(7_{00}-6_{00}\right)$ spectra observed toward $\mathrm{J} 18354 \mathrm{~S}$, while Figure 11 shows the paratransitions $\mathrm{H}_{2} \mathrm{CO} 3_{03}-2_{02}$ at $218.222 \mathrm{GHz}$ and $3_{22}-2_{21}$ at $218.475 \mathrm{GHz}$ spectra observed around both cores (J18354S 

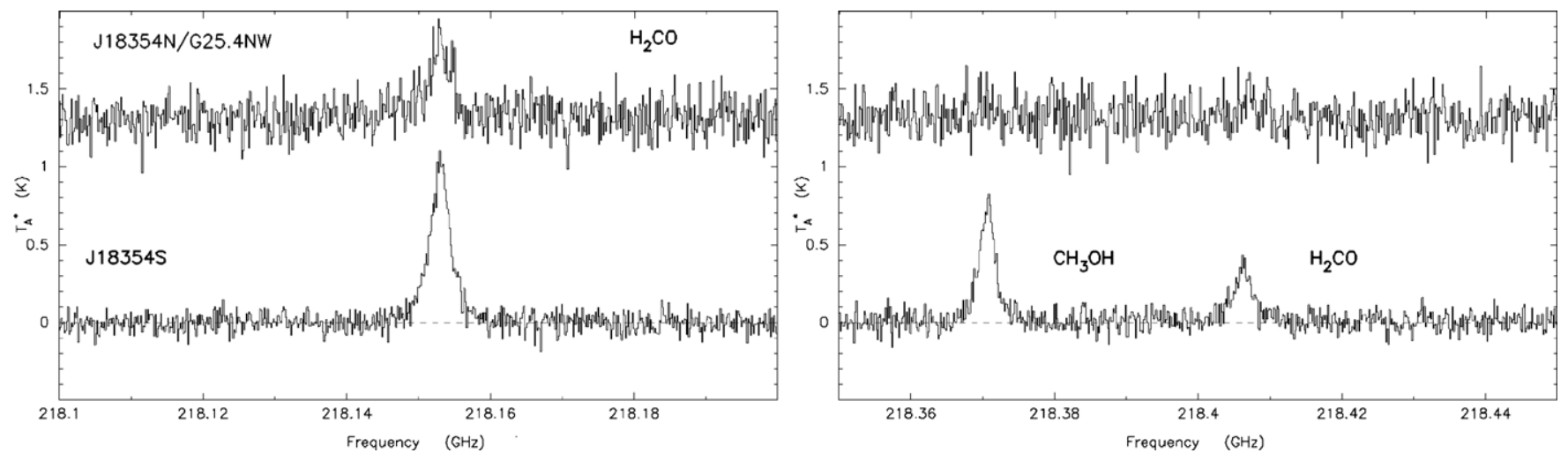

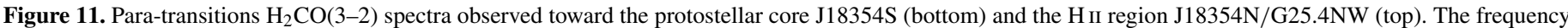
has been redshifted by $0.07 \mathrm{GHz}$ (corresponds to $96.7 \mathrm{~km} \mathrm{~s}^{-1}$ ). The line at $218.37 \mathrm{GHz}$ is the $\mathrm{CH}_{3} \mathrm{OH}-\mathrm{E}\left(4_{20}-3_{10}\right)$ transition. The temperature scale is $T_{A}^{*}$.

and J18354N). Liu et al. (2011) also published an SMA map of $\mathrm{CH}_{3} \mathrm{OH}\left(5_{23}-4_{13}\right)$. The detection of these lines indicates that part of the gas in $\mathrm{J} 18354 \mathrm{~S}$ is being heated up by the protostar, since the abundances of $\mathrm{H}_{2} \mathrm{CO}$ and $\mathrm{CH}_{3} \mathrm{OH}$ are very sensitive to temperature (Hatchell et al. 1998). These molecular species are normally frozen in dust grains; an appreciable gas-phase abundance is only encountered when a young star heats up the ambient medium. Moreover, both molecules are easily destroyed by a strong UV radiation field, like that seen in the northern $\mathrm{H}$ II region, G25.4NW, where notably we find rather weak $\mathrm{H}_{2} \mathrm{CO}(3-2)$ emission and virtually no $\mathrm{CH}_{3} \mathrm{OH}-\mathrm{E}\left(4_{20}-3_{10}\right)$ emission (Figure 11, upper spectrum). According to the $\mathrm{LVG}$ analysis result of Mangum \& Wootten (1993), the ratio $T_{A}^{*}\left(3_{03}-2_{02}\right) / T_{A}^{*}\left(3_{22}-2_{21}\right)$ depends on both gas density and kinetic temperature. Our measured ratio is $T_{A}^{*}\left(3_{03}-2_{02}\right) / T_{A}^{*}\left(3_{22}-2_{21}\right)=3.3$, suggesting that the gas temperature in $\mathrm{J} 18354 \mathrm{~S}$ is $\gtrsim 75 \mathrm{~K}$ when the gas density is in the range $10^{5}-10^{7} \mathrm{~cm}^{-3}$. Thus, this source could be in the hot core phase (Churchwell 2002), or a hot core is developing.

Both our data and the SMA maps show that the size of the warm gas traced by $\mathrm{CH}_{3} \mathrm{OH}$ is as large as that of the cold dust traced by submillimeter continuum emission. A possible explanation is that the protostar IRS1a is forming on the surface of the dust core, heating up the surrounding gas. The visual extinction of IRS1a was estimated to be $A_{\mathrm{v}}=35$, while the total column density of the dust core is as high as $5 \times 10^{23} \mathrm{~cm}^{-2}$, corresponding to $A_{\mathrm{v}} \sim 530\left(A_{\mathrm{v}}=N_{\mathrm{H}} / 0.94 \times 10^{21}\right.$; Frerking et al. 1982). Thus, the protostar and the hot/warm gas should be in the front envelope of the dust core.

The $\mathrm{C}^{18} \mathrm{O}(2-1)$ map is dramatically different from our SCUBA dust maps. Figure 12 shows contours of the integrated line intensity of $\mathrm{C}^{18} \mathrm{O}(2-1)$ overlaid on to an $850 \mu \mathrm{m}$ image. The southern $\mathrm{C}^{18} \mathrm{O}$ core is not as strong as the northern core, indicating a certain degree of $\mathrm{CO}$ depletion. Also, the southern $\mathrm{C}^{18} \mathrm{O}$ core is not particularly compact, suggesting that the $\mathrm{C}^{18} \mathrm{O}$ may be optically thick in the high-density core region.

Carolan et al. (2009) have used $\mathrm{C}^{17} \mathrm{O}$ and $\mathrm{C}^{18} \mathrm{O}(2-1)$ data to estimate the $\mathrm{H}_{2}$ column density. They find it to be a factor of 10 lower than that derived from the dust mass. For comparison, we also do the same calculation for the northern core and find that the depletion factor is about 1, i.e., no CO depletion at all. This is consistent with the fact that most of the CO gas will be released into the gas phase in a fully developed $\mathrm{H}$ II region.

In summary, there are at least two gas components associated with the J18354S core, a hot gas component with a temperature of more than $75 \mathrm{~K}$ that envelopes the protostar and a much

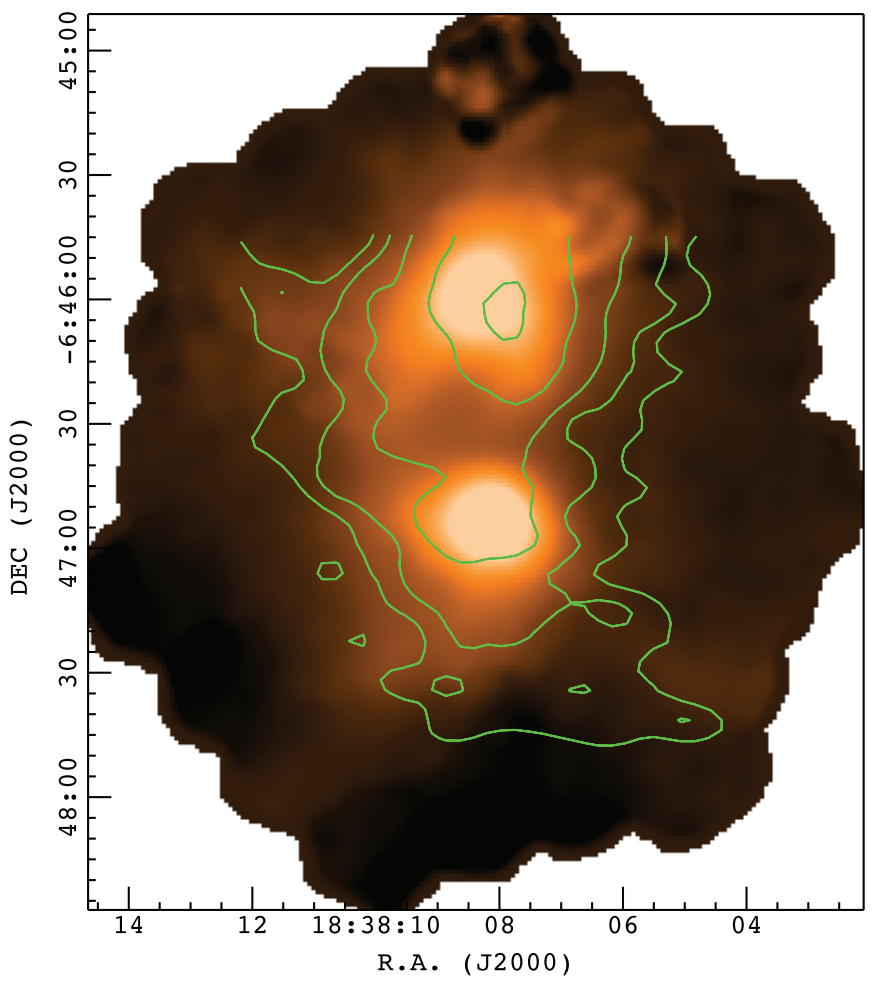

Figure 12. Contours of $\mathrm{C}^{18} \mathrm{O}(2-1)$ integrated line intensity overlaid on top of the SCUBA $850 \mu \mathrm{m}$ image of cores J18354N and J18354S. The contour levels are $7.5,10.6,15,21.2$, and $30 \mathrm{~K} \mathrm{~km} \mathrm{~s}^{-1}$ on the $T_{A}^{*}$ scale.

(A color version of this figure is available in the online journal.)

colder central region $(T \sim 18 \mathrm{~K})$ where $\mathrm{CO}$ is depleted. Highresolution interferometric maps with SMA (Liu et al. 2011) show that J18354S is indeed resolved into several dust clumps, with the brightest clump offset from IRS1a by 1 1.6. It is therefore possible that the majority of the gas is still cold and more stars will form out of the massive submillimeter core, J18354S.

\section{DISCUSSION}

\subsection{The Evolutionary Status of J18354S}

The analysis in the previous sections suggests that J18354S, the southern SCUBA core in the JCMT 18354-0649 region, contains a massive young star $\left(6-12 M_{\odot}\right)$, although the majority of the luminosity is in the submillimeter regime. We label the NIR/MIR object near the center of the J18354S core IRS1a; this object appears to be a protostar in a very early stage of 
evolution and may be a counterpart of a low-mass Class 0 or Class I protostar.

Both infall and outflow motions are seen in the gas envelope that surrounds this source. There is evidence for a collimated jet extending to the southwest in the MIR images, although this requires confirmation with shorter wavelength and/or higher resolution imaging. The low MIR emission at 24 and $75 \mu \mathrm{m}$ and lack of radio continuum thermal emission suggest that IRS1a is in the early stages of heating up the surrounding gas. According to the composite models of Keto (2007), it is in the "hypercompact H II region" phase, which is the evolutionary phase associated with the formation of massive stars of type B and earlier, when the stars are both hot enough to ionize an H II region around the star and yet are still growing by accretion. The models further suggest an evolutionary sequence driven by the increasing ionization of a star growing by accretion. The YSO IRS1a in J18354S is a good example of such an object. It is still actively accumulating material and thus will become more massive as it evolves. Our SED fitting provides a lower limit to the stellar mass of IRS1a of roughly $6-12 M_{\odot}$. Since IRS1a/J18354S is at the very earliest stages of MSF, if the accretion does indeed continue then the star could grow into a much more massive object. Our combined near-, mid-, and far-IR data suggest that an accretion scenario could explain the formation of such a massive star.

\subsection{The Distance to J18354S}

The distance to $\mathrm{J} 18354 \mathrm{~S}$ is a major uncertainty in this work. The radial velocity of molecular spectral lines peaks at $96.7 \mathrm{~km} \mathrm{~s}^{-1}$, which suggests that it is at $5.7 \mathrm{kpc}$ or $9.6 \mathrm{kpc}$ according to the Galactic rotation curve. We still need to solve the well-known (near-far) distance ambiguity. More data are available for the northern $\mathrm{H}$ II region, G25.4NW, whose radial velocity from molecular lines (e.g., $\mathrm{C}^{18} \mathrm{O} J=2-1$ ) is similar to that of J18354S. The morphology of the dust and $\mathrm{C}^{18} \mathrm{O}$ maps (Figures 1 and 12) suggests that J18354S is located in the same large cloud as $\mathrm{G} 25.4 \mathrm{NW}$. The spectral profiles of ${ }^{13} \mathrm{CO}$ and $\mathrm{C}^{18} \mathrm{O}$ show a smooth change from J18354S to G25.4NW, indicating a kinematic connection between them. Hence, we can use G25.4NW to determine the distance of J18354S.

Downes et al. (1980) assign a far-side distance of $9.6 \mathrm{kpc}$ to the molecular cloud G25.4-0.14 (G25.4NW in our map), based on its $\mathrm{OH}$ absorption features at $\sim 95 \mathrm{~km} \mathrm{~s}^{-1}$. Solomon et al. (1987) also suggest a far-side distance of $11.5 \mathrm{kpc}$, based on a scale height method. The papers of Lester et al. (1985), Churchwell et al. (1990), and Giveon et al. (2007) all use the farside distance of $9.8 \mathrm{kpc}$ for G25.4NW. Churchwell et al. (1990) estimate a far-IR luminosity of $L_{\mathrm{IR}}=10^{6.238} L_{\odot}$, indicating the presence of an O4 or O5 star. However, Giveon et al. (2007) found that the radio-derived spectral type, which is based on the free-free emission and does not suffer from dust extinction, is O7-O8. This suggests that the stars in G25.4NW are not as hot as indicated by the far-IR data. Such a discrepancy would disappear if the near-side distance of $5.7 \mathrm{kpc}$ was used, where the far-IR luminosity would correspond to an O9 star and the radio-derived spectral type would be 09.5 , using the stellar model atmospheres of Sternberg et al. (2003).

For the southern core J18354S, our SED modeling also shows that the near-side distance results in a lower temperature for the central star IRS1a, which is consistent with the low midIR emission at $24 \mu \mathrm{m}$ and $70 \mu \mathrm{m}$. If the far-side distance of $9.6 \mathrm{kpc}$ is used, the SED modeling (Figure 7(a)) would indicate a central star with a stellar mass of 10-20 $M_{\odot}$, and a temperature of 4000-10,000 K, equivalent to a B2 star or earlier. Such a star should have strong $24 \mu \mathrm{m}$ emission.

It is therefore possible that the distance of $\mathrm{G} 25.4 \mathrm{NW}$ and $\mathrm{J} 18354 \mathrm{~S}$ has been mis-determined. Distances derived from $\mathrm{OH}$ absorption features and via the scale height method carry large uncertainties. A wrong distance was derived in the case of the nearby $\mathrm{H}$ II region G25.4-0.2 (W42 in our map). It has a radial velocity of $65 \mathrm{~km} \mathrm{~s}^{-1}$ and was assigned a far-side distance of $13.5 \mathrm{kpc}$ by Downes et al. (1980) and Solomon et al. (1987) (distance $=13.2 \mathrm{kpc})$. This value was questioned by Lester et al. (1985), based on the low extinction derived from their S[III] $\lambda 9532$ data. Using the $\mathrm{H} 86 \alpha$ spectral line, Lester et al. (1985) argued that the near-side distance of $4.6 \mathrm{kpc}$ should be used for W42. A similar situation could also be applied to J18354S and G25.4NW. We plan to use near-IR spectroscopy to determine the spectral type of the massive stars in G25.4NW, and thereby to solve the distance problem; this will be the content of a future paper on $\mathrm{G} 25.4 \mathrm{NW}$.

We note that, assuming a near-side distance of $5.7 \mathrm{kpc}$ for J18354S, Wu et al. (2005) find that the submillimeter core has an angular size (diameter) of $10^{\prime \prime}$, corresponding to $0.28 \mathrm{pc}$. Carolan et al. (2009) estimate that the CO freezeout radius is $\sim 20^{\prime \prime}$, corresponding to $0.5 \mathrm{pc}$. Both of these physical scales are consistent with the sizes of most massive cores (e.g., Williams et al. 2000). If J18354S was at a distance of $9.6 \mathrm{kpc}$, the core size would be $0.47 \mathrm{pc}$ and the $\mathrm{CO}$ freezeout radius would be $0.84 \mathrm{pc}$; these are larger than most known cores. In summary, the near-side distance of $5.7 \mathrm{kpc}$ results in more reasonable physical parameters for J18354S.

\section{SUMMARY AND CONCLUSION}

Near-infrared images and photometry have been obtained for the massive SCUBA core J18354S. An extremely red object has been discovered embedded within the core. Modeling of the SED of this object suggests that it is a massive protostar, with a mass of 6-12 $M_{\odot}$, and that this object is still actively accreting mass inside the submillimeter core. The YSO and core probably represent a very early phase of MSF.

The core itself has been observed at mid-IR and far-IR wavelengths. By combining CSO and JCMT data at 350, 450, and $850 \mu \mathrm{m}$, we derive a total gas mass of $670 M_{\odot}$. The core is massive but also cold; we derive a spectral index, $\beta$, of 2.3 and a dust temperature, $T_{\mathrm{d}}$, of $\sim 18 \mathrm{~K}$.

Our near-IR observations show that at least two near-IR protostars are forming inside the core: thus, there could possibly be a cluster forming within $\mathrm{J} 18354 \mathrm{~S}$.

The low dust temperature at the core, low mid-IR emission, and lack of thermal radio continuum emission suggest that the core $\mathrm{J} 18354 \mathrm{~S}$ is in a very early stage of heating up the surrounding gas. It may be in the "hypercompact H II region" phase of MSF. Several transitions of $\mathrm{CH}_{3} \mathrm{OH}$ and $\mathrm{H}_{2} \mathrm{CO}$ have been detected toward $\mathrm{J} 18354 \mathrm{~S}$, and the gas temperature is estimated to be higher than $75 \mathrm{~K}$, suggesting a hot core is developing around the embedded protostar, though the majority of the gas in the core is still cold.

M.Z. acknowledges the support from NSFC grant Y011231001 and also from the "Hundred-talent program" of the Chinese Academy of Sciences. Y. Wu is grateful for the support of grants 10733030 and 10873019 of NSFC. The United Kingdom Infrared Telescope is operated by the Joint Astronomy Centre (JAC) on behalf of the U.K. Science and Technology Facilities Council (STFC). The $H$-, $K$-, and $L^{\prime}$-band imaging 
reported here were obtained as part of the UKIRT Service Programme. We thank Dr. Watson Varricatt at the JAC for help with near-IR photometry. The JCMT is also operated by the Joint Astronomy Centre on behalf of the U.K. STFC, the National Research Council Canada, and the Netherlands Organisation for Scientific Research. Caltech Submillimeter Observatory is supported through NSF grant AST-0540882. This work is based in part on observations made with the Spitzer Space Telescope, which is operated by the Jet Propulsion Laboratory, California Institute of Technology, under a contract with NASA. Support for this work was provided by NASA through an award issued by JPL/Caltech for the GLIMPSE360 project (B.W.). We thank the GLIMPSE team (PI: E. Churchwell) for making the IRAC images available on the internet. We also thank the anonymous referee whose comments helped improve the paper.

\section{REFERENCES}

Allen, L. E., Calvet, N., D’Alessio, P., et al. 2004, ApJS, 154, 363

Andre, P., Ward-Thompson, D., \& Barsony, M. 2000, in Protostars and

Planets IV, ed. V. Mannings, A. P. Boss, \& S. S. Russell (Tucson, AZ: Univ. Arizona Press), 59

Aspin, C., \& Sandell, G. 1997, MNRAS, 289, 1

Avery, L. W., \& Chiao, M. 1996, ApJ, 463, 642

Benjamin, R. A., Churchwell, E., Babler, B. L., et al. 2003, PASP, 115, 953

Bergin, E. A., Snell, R. L., \& Goldsmith, P. F. 1996, ApJ, 460, 343

Berné, O., Fuente, A., Goicoechea, J. R., et al. 2009, ApJ, 706, L160

Beuther, H., Churchwell, E. B., McKee, C. F., \& Tan, J. C. 2007, in Protostars and Planets V, ed. B. Reipurth, D. Jewitt, \& K. Keil (Tucson, AZ: Univ. Arizona Press), 165

Beuther, H., \& Shepherd, D. 2005, in Cores to Clusters: Star Formation with Next Generation Telescopes, ed. M. S. N. Kumar, M. Tafalla, \& P. Caselli (New York: Springer), 105

Beuther, H., Schilke, P., Menten, K. M., et al. 2002, ApJ, 566, 945

Beuther, H., \& Steinacker, J. 2007, ApJ, 656, L85

Bonnell, I. A., Bate, M. R., \& Zinnecker, H. 1998, MNRAS, 298, 93

Brand, J., Cesaroni, R., Palla, F., \& Molinari, S. 2001, A\&A, 370, 230

Cardelli, J. A., Clayton, G. C., \& Mathis, J. S. 1989, ApJ, 345, 245

Carey, S. J., Noriega-Crespo, A., Price, S. D., et al. 2005, BAAS, 37, 1252

Carolan, P. B., Khanzadyan, T., Redman, M. P., et al. 2009, MNRAS, 400, 78

Churchwell, E. 2002, ARA\&A, 40, 27

Churchwell, E., Walmsley, C. M., \& Cesaroni, R. 1990, A\&AS, 83, 119

Cyganowski, C. J., Whitney, B. A., Holden, E., et al. 2008, AJ, 136, 2391

Davis, C. J., Kumar, M. S. N., Sandell, G., et al. 2007, MNRAS, 374, 29

Davis, C. J., Scholz, P., Lucas, P. W., \& Adamson, A. 2008, MNRAS, 387, 954

Davis, C. J., Varricatt, W. P., Todd, S. P., \& Ramsay Howat, S. K. 2004, A\&A, 425,981

Dionatos, O., Nisini, B., Garcia Lopez, R., et al. 2009, ApJ, 692, 1

Dowell, C. D., Allen, C. A., Babu, R., et al. 2003, Proc. SPIE, 4855, 73

Downes, D., Wilson, T. L., Bieging, J., \& Wink, J. 1980, A\&AS, 40, 379
Fazio, G. G., Hora, J. L., Allen, L. E., et al. 2004, ApJS, 154, 10

Frerking, M. A., Langer, W. D., \& Wilson, R. W. 1982, ApJ, 262, 590

Friesen, R. K., Johnstone, D., Naylor, D. A., \& Davis, G. R. 2005, MNRAS, 361,460

Gibb, A. G., Richer, J. S., Chandler, C. J., \& Davis, C. J. 2004, ApJ, 603, 198

Giveon, U., Richter, M. J., Becker, R. H., \& White, R. L. 2007, AJ, 133, 639

Goldsmith, P. F., Bergin, E. A., \& Lis, D. C. 1997, ApJ, 491, 61

Greve, T. R., Papadopoulos, P. P., Gao, Y., \& Radford, S. J. E. 2009, ApJ, 692, 1432

Hatchell, J., Thompson, M. A., Millar, T. J., \& Macdonald, G. H. 1998, A\&ASS, 133,29

Johnstone, D., \& Bally, J. 1999, ApJ, 510, L49

Jorgensen, J. K., Johnstone, D., van Dishoeck, E. F., \& Doty, S. D. 2006, A\&A, 449,609

Keto, E. 2007, ApJ, 666, 976

Lahuis, F., \& van Dishoeck, E. F. 2000, A\&A, 355, L699

Leong, M., Peng, R., Houde, M., et al. 2006, Proc. SPIE, 6275, 62750

Lester, D. F., Dinerstein, H. L., Werner, M. W., et al. 1985, ApJ, 296, 565

Lis, D. C., \& Menten, K. M. 1998, ApJ, 507, 794

Lis, D. C., Serabyn, E., Keene, J., et al. 1998, ApJ, 509, 299

Liu, T., Wu, Y., Zhang, Q., Ren, Z., Guan, X., \& Zhu, M. 2011, ApJ, 728, 91

Maercker, M., Burton, M. G., \& Wright, C. M. 2006, A\&A, 450, 253

Mangum, J. G., \& Wootten, A. 1993, ApJS, 89, 123

Martin-Pintado, J., Bachiller, R., \& Fuente, A. 1992, A\&A, 254, 315

McKee, C. F., \& Tan, J. C. 2003, ApJ, 585, 850

Meyer, M. R., Calvet, N., \& Hillenbrand, L. A. 1997, AJ, 114, 288

Neufeld, D. A., Nisini, B., Giannini, T., et al. 2009, ApJ, 706, 170

Ossenkopf, V., \& Henning, T. 1994, A\&A, 291, 943

Phillips, J. P., \& Ramos-Larios, G. 2008, MNRAS, 391, 1527

Ramsay Howatt, S. K., Todd, S. P., Leggett, S. K., et al. 2004, Proc. SPIE, 5492, 1160

Rathborne, J. M., Jackson, J. M., Chambers, E. T., et al. 2005, ApJ, 630, 181

Robitaille, T. P., Whitney, B. A., Indebetouw, R., \& Wood, K. 2007, ApJS, 169, 328

Robitaille, T. P., Whitney, B. A., Indebetouw, R., Wood, K., \& Denzmore, P. 2006, ApJS, 167, 256

Schnee, S., Enoch, M., Noriega-Crespo, A., et al. 2010, ApJ, 708, 127

Shepherd, D. S., Nürnberger, D. E. A., \& Bronfman, L. 2004, ApJ, 602, 850

Shepherd, D. S., Povich, M. S., Whitney, B. A., et al. 2007, ApJ, 669, 464

Smith, M. D. 1991, MNRAS, 252, 378

Solomon, P. M., Rivolo, A. R., Barrett, J., \& Yahil, A. 1987, ApJ, 319, 730

Sternberg, A., Hoffmann, T. L., \& Pauldrach, A. W. A. 2003, ApJ, 599, 1333

Tokunaga, A. T. 2000, in Allen's Astrophysical Quantities, ed. A. N. Cox (4th ed.; New York: Springer), 143

Whitney, B. A., Wood, K., Bjorkman, J. E., \& Wolff, M. J. 2003, ApJ, 591, 1049

Williams, J., Blitz, L., \& McKee, C. 2000, in Protostars and Planets IV, ed. V. Mannings, A. P. Boss, \& S. S. Russell (Tucson, AZ: Univ. Arizona Press), 97

Williams, J. S., Fuller, G. A., \& Sridharan, T. K. 2004, A\&A, 417, 115

Wu, Y., Zhang, Q., Yu, W., et al. 2006, A\&A, 450, 607

Wu, Y., Zhu, M., Wei, Y., et al. 2005, ApJ, 628, L57

Ybarra, J. E., \& Lada, E. A. 2009, ApJ, 695, L120

Zhu, M., Papadopoulos, P. P., Xilouris, E. M., Kuno, N., \& Lisenfeld, U. 2009, ApJ, 706, 941

Zhu, M., Seaquist, E. R., \& Kuno, N. 2003, ApJ, 588, 243 